\title{
The Layout of Iso-orientation Domains in Area 18 of Cat Visual Cortex: Optical Imaging Reveals a Pinwheel-like Organization
}

\author{
Tobias Bonhoeffer ${ }^{1}$ and Amiram Grinvald 1 ,2,3,a \\ 'Laboratory of Neurobiology, The Rockefeller University, New York, New York 10021, '2Department of Neurobiology, The \\ Weizmann Institute of Science, Rehovot, 7600, Israel, and ${ }^{3}$ BBM Research Division, Yorktown Heights, New York 10598
}

In this study we used optical imaging based on activitydependent intrinsic signals to determine the distribution of cells responding to gratings of various orientations moving in different directions in area 18 of cat visual cortex. To test directional-selective clustering of neurons, we compared cortical activity maps obtained by stimulation with two gratings of identical orientation but moving in opposite directions. We found those maps to be almost identical, suggesting that neurons are not notably clustered into directionality columns. We also compared activity maps obtained with gratings of different orientations. Each of the orientation maps was similar to the 2-deoxyglucose maps previously reported. Having compiled the information obtained from the different orientations into one "orientation preference map," we found, in contrast to earlier reports, that iso-orientation domains are not elongated parallel bands but are small patches organized in "pinwheels" around points that we refer to as "orientation centers." We furthermore show that the only locations at which orientation preference changes rapidly are these orientation centers and not lines or loops. In addition, this report clarifies that our observations on the functional architecture of cat area 18, although at first sight at variance with earlier observations, are actually fully consistent with them. We therefore propose that in cat visual cortex pinwheel-like patterns of orientation preference form an irregular mosaic of modular units with an average density of $\mathbf{1 . 2}$ pinwheels per square millimeter.

IKey words: visual cortex, functional architecture, cortical map, optical imaging, area 18, cat]

Neurons in the visual cortex transform visual input arriving via the lateral geniculate body into patterns of electrical activity corresponding to crucial attributes of the visual scene. The intricate geniculocortical and intrinsic cortical connections endow cortical cells with remarkably selective response properties. For example, single cortical cells respond selectively to different types of visual stimuli like edges of lines of a given orientation, specific directions of motion, or certain ocular disparities. Hubel and Wiesel (1963) found that visual cortical cells with similar

\footnotetext{
Received Nov. 13, 1992; revised Mar. 25, 1993; accepted Apr. 7, 1993.

We thank T. N. Wiesel, C. D. Gilbert, and D. Y. Ts'o for their useful suggestions regarding these experiments, D.-S. Kim, D. Shoham, and D. Malonek for their help in some of the experiments and for their constructive criticism, $S$. Löwel for helpful discussions and comments on the manuscript, and D. Y. Ts'o, E. Ratzlaff, and $K$. Christian for their excellent support in hardware and software matters.

Correspondence should be addressed to Tobias Bonhoeffer, Max Planck Institute for Psychiatry, Am Klopferspitz 18A, 82152 München-Martinsried, Germany.

aPresent address: The Weizmann Institute of Science, Rehovot, 7600, Israel.

Copyright (C) 1993 Society for Neuroscience $0270-6474 / 93 / 134157-24 \$ 05.00 / 0$
}

response properties are frequently clustered together, forming modules that span the entire depth of the cortex from the pia to the white matter. Tangentially, the cortex is organized such that response properties change gradually, thereby forming representations of the environment on the cortical surface, the socalled cortical maps. Such maps have been demonstrated for several parameters of the visual space, the most prominent being the retinotopic, the ocular dominance, and the orientation maps.

One general principle in these maps is that neighborhood relationships are preserved as far as possible. For the retinotopic map, for example, images of the environment are projected onto the retina and relayed to the lateral geniculate body and the visual cortex in an orderly manner. This causes neighboring points of the visual scene to be represented at nearby locations in the primary visual cortex. Thus, in a retinotopic map, neighborhood is defined geometrically. Alternatively, neighborhood can be understood perceptually: in an orientation map, for example, the orientation of a line is the parameter according to which cells are grouped together. In this context, neighborhood is defined in an abstract manner: two similar orientations will be processed in locations close together in space. Since this neighborhood-preserving mapping is a principle used throughout the cerebral cortex, it seems to be of great importance for the brain to organize some cortical representations in this fashion. It is therefore of considerable interest to learn more about the precise way in which visual attributes of retinal images are mapped onto the cortex. A deeper comprehension of cortical maps is thus essential not only from a descriptive point of view, but also to gain an understanding of the principles underlying development and/or function of the cerebral cortex.

Until recently it was only possible to map the architecture of cortical functional domains in two ways. The first-electrophysiological - approach uses multiple electrode penetrations to determine response properties from single cells in various cortical locations (Tusa et al., 1978, 1979; Kaas et al., 1979; Swindale et al., 1987). This approach is very tedious and has a limited spatial resolution: 2500 electrode penetrations are required in order to map a field of $5 \times 5 \mathrm{~mm}$ with a resolution of $100 \mu \mathrm{m}$. Therefore, the accuracy that can be achieved with this method is rather limited in practice. The second approach is to use the radioactively labeled sugar analog 2-deoxyglucose (2-DG) and label the neurons that have a higher metabolic demand in response to a certain stimulus (Sokoloff, 1977; Hubel et al., 1978). This technique has a very good spatial resolution, it is applicable to the entire brain but only permits the mapping of activity cvoked by onc particular stimulus.' ${ }^{\prime}$ Furthermorc, the map can

If one radioactive isotope is used. A maximum of two different stimuli can be mapped by using labeling to 2-DG with two different radioactive isotopes (Livingstone and Hubel, 1981; Friedmann et al., 1987; Redies et al., 1990). 
only be obtained post-mortem, since the animal has to be killed to visualize the distribution of the radioactive sugar analog. Subsequent electrophysiological or anatomical measurements, on a formerly mapped cortical patch, are therefore impossible with the 2-DG method.

To study architecture and function of cortex, an alternative technique has been developed that images the neuronal activity in the living brain. This technique, based on voltage-sensitive dyes, was used to study the frog optic tectum (Grinvald et al., 1984), the rat somatosensory cortex (Orbach et al., 1985), the functional architecture of the primary visual cortex of the macaque monkey (Blasdel and Salama, 1986; Lieke et al., 1993), the salamander olfactory bulb (Kauer, 1988), the cerebellum (Kim et al., 1989), and the rat cortex (Orbach et al., 1985; London et al., 1989). Subsequently, it has been shown how, provided that time resolution is not important, high-resolution mapping of the functional architecture can even be obtained without voltage-sensitive dyes by monitoring activity-dependent changes of the optical properties intrinsic to the tissue (Grinvald et al., 1986, 1991; Frostig et al., 1990; Ts'o et al., 1990; Bonhoeffer and Grinvald, 1991; Bartfeld and Grinvald, 1992). This technique makes use of the fact that active regions of cortex consume oxygen that has to be provided by the hemoglobin molecules flowing through the microvasculature in the brain. Thus, in activated cortical regions, the red oxyhemoglobin is converted into reddish blue deoxyhemoglobin. This causes activated cortical regions to have higher absorption of red light compared to less active areas (Grinvald et al., 1986; Frostig et al., 1990). To obtain functional maps one acquires in vivo a high-resolution image from a brain area activated by a specific stimulus. When this image is compared to a second image from the same area in a nonactivated state, the comparison selectively reveals the very small optical changes in the cortical regions that were activated by the respective stimulus. Therefore, although the activity-dependent light intensity changes at the optimal wavelength for optical mapping constitute 1-2 parts in 1000 of the reflected light intensity, high-resolution functional maps can be obtained. Since this procedure is carried out in the living brain it can be repeated with as many different stimuli as desired. Consequently, with this method a cortical area can be investigated in much more detail than with the 2-DG method, in which only the response to one or two stimuli can be mapped. Furthermore, due to the fact that the animal does not have to be killed merely to obtain the map, electrical recordings and anatomical investigations such as tracer injections can be guided by the detailed optical map previously obtained. It is clear, however, that the 2-DG technique offers different advantages somewhat complementary to optical imaging: it provides a laminar resolution across the different cortical layers and the activity in the entire brain can be imaged, not just the regions under a cranial window.

In this study we used "optical imaging based on intrinsic signals" to investigate the functional architecture in cat area 18 . The functional architecture of the visual cortices in cat and monkey has been extensively explored and vigorously debated during the past two or three decades. Hubel and Wiesel performed electrode penetrations tangential to the cortical surface and found gradual changes of some response properties (Hubel and Wiesel, 1962). For example, orientation preference and ocular dominance of single cells changed continuously with the distance that the electrode had traveled parallel to the cortical surface. From such observations they concluded that both ocular dominance and orientation preference are organized in elongated bands. This view was later corroborated by the anatomical demonstration of ocular dominance bands using transneuronal labeling (Wiesel et al., 1974). Iso-orientation bands were also demonstrated using the 2-DG technique (Hubel et al., 1978). The view of parallel bands of iso-orientation domains was, however, challenged, mainly in theoretical articles (von Seelen, 1970; Braitenberg and Braitenberg, 1979). These articles showed that an alternative arrangement, a radial organization of iso-orientation domains, would be consistent with the reported electrophysiological findings. This controversy was not resolved by two apparently contradictory experimental studies: Swindale et al. (1987) conducted a very extensive electrophysiological mapping study of cat area 18 and concluded that the radial organization prevailed. In contrast, Löwel et al. (1987), using the 2-DG technique, concluded that iso-orientation domains in cat area 17 are parallel "beaded bands." Earlier studies in the monkey using optical imaging (Blasdel and Salama, 1986; Blasdel, 1989; Ts'o et al., 1990) did not conclusively decide between the "banded" and the "radial" theory.

A second controversial question was whether preferred direction of motion is a further parameter according to which the visual cortex is parcelated. While Hubel and Wiesel $(1962,1965)$ have shown cells with both sharp orientation tuning and clear directional selectivity, they did not report clustering of cells with similar direction preferences. However, three other groups (Payne et al., 1980; Tolhurst et al., 1981; Swindale et al., 1987) reported that their electrophysiological mapping studies did show significant clustering and yielded directional maps. Since the techniques employed in those earlier studies have the limitations already mentioned, we decided to use optical imaging based on intrinsic signals to resolve the above two controversial issues.

\section{Materials and Methods}

For imaging based on intrinsic signals we have used the procedures that where developed by Grinvald, Lieke, Frostig, Ratzlaff, Ts'o, Bonhoeffer, Shoham, and Malonek. Here we describe some previously unpublished details.

Animals. Eleven cats were initially anesthetized with ketamine hydrochloride (10-20 mg/kg, i.m.), supplemented in some cases by $x y-$ lazine hydrochloride $(2.5 \mathrm{mg} / \mathrm{kg}$, i.m.) followed by either a barbiturate (sodium pentothal, $20 \mathrm{mg} / \mathrm{kg}$, i.v.) or a halothane $/ \mathrm{N}_{2} \mathrm{O}$ anesthesia $(70 \%$ $\mathrm{N}_{2} \mathrm{O}, 30 \% \mathrm{O}_{2}$ with $0.2-2 \%$ halothane). A tracheotomy was performed and EEG electrodes were implanted. To prevent eye movements, the animal was paralyzed (succinylcholine hydrochloride, $20 \mathrm{mg} / \mathrm{kg} / \mathrm{hr}$, or hexacarbacholinbromide, $0.3-1 \mathrm{mg} / \mathrm{kg} / \mathrm{hr}$, i.v.). After paralysis the animal was artificially respirated. To monitor the condition of the animal, EEG, ECG, expired $\mathrm{CO}_{2}$, and temperature were controlled throughout the experiment. To focus the eyes on the monitor screen, contact lenses (in some cases with an artificial pupil of $3 \mathrm{~mm}$ ) were fitted to the cat's eyes with the aid of a retinoscope.

Optical chamber. The skull of the cat was opened above area 18 or 17 by opening two half-circled holes whose "centers" lay between Horsley-Clarke A5 and A0 (depending on the cortical area to be investigated) and $0.5-1 \mathrm{~mm}$ lateral of the midline. A stainless steel chamber was cemented onto the skull, the dura was removed, and the chamber was filled with silicone oil and finally sealed with a round glass coverslip. To facilitate electrical recordings through the sealed chamber, in some instances a rubber gasket was glued into a 3-mm-diameter hole made in the round coverglass. Without the sealed chamber, cortical movements duc to heartbeat and respiration severely hampered optical recording. The sealed chamber also proved to be a significant improvement for electrical recordings, which were very stable over long periods of time.

CCD camera and optics. The basic experimental setup is depicted in Figure 1. The surface of the cortex was illuminated by two adjustable light guides. These guides were attached to a Zeiss tungsten-halogen lamp. The light was passed through interference filters of different wave- 


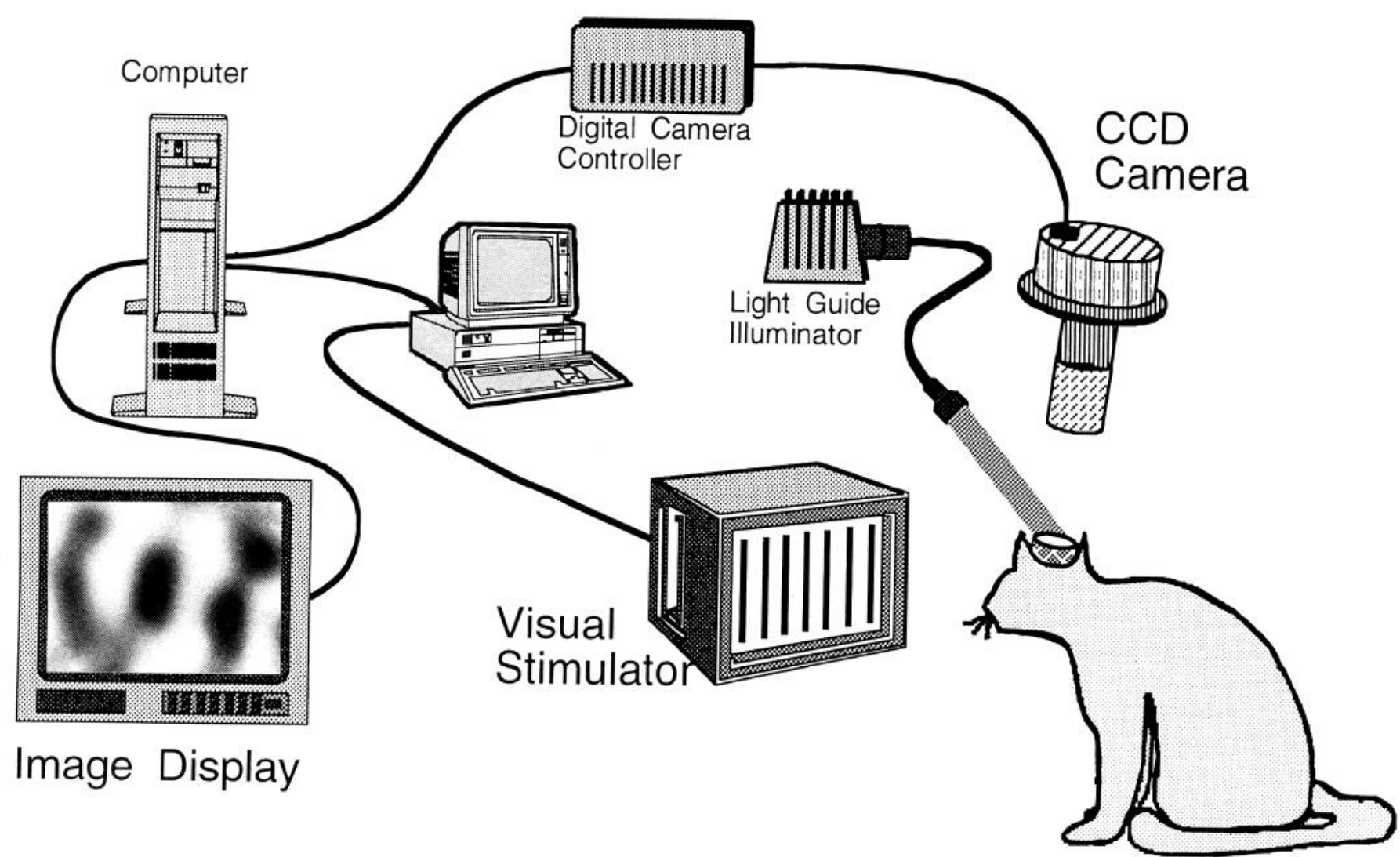

Figure 1. A setup for optical imaging of functional maps in vivo. Digital CCD images are taken from the cortex of the cat, exposed in a sealed, oil-filled chamber. The cortex is illuminated with light of $605 \mathrm{~nm}$ wavelength. The images are acquired with the CCD camera while the cat is visually stimulated with gratings moving on a CRT screen. These pictures are digitized in a camera controller that transfers the data to the computer controlling the entire experiment. Functional maps can then be analyzed on this computer and shown on an image display monitor. To determine the quality of the maps during the imaging sessions the data can be sent to a second computer for detailed, quasi-on-line analysis.

lengths. The filter most often used for visualizing the surface of the cortex, and its vascular pattern had a transmission maximum at $540 \pm$ $30 \mathrm{~nm}$, whereas the filter used for the optical imaging was a $605 \pm 10$ $\mathrm{nm}$ filter. This wavelength was chosen since it coincides with the peak of the difference spectrum between oxyhemoglobin and deoxyhemoglobin: one maximizes the contribution of oximetry signals relative to other intrinsic signals (Frostig et al., 1990). To achieve nearly uniform illumination of cortex, the two adjustable light guides could be aimed separately at the cortex.

A slow-scan CCD camera (Photometrics Ltd., Tucson, AZ) was used for the imaging based on intrinsic signals as previously described (Ts'o et al., 1990). The camera was mounted above the optical chamber and was aimed so that its optical axis was perpendicular to the cortical surface. The camera provided 12 bit digitized images of the cortex. These pictures had a spatial resolution of $192 \times 144$ pixels $(2 \times 2$ binning, prior to the digitization of the camera output, was used) and were transferred to a Microvax III workstation operating under Ultrix or VMS. The root-mean-square (RMS) noise obtained by this camera configuration was 1 part in 1400 .

To minimize artifacts caused by blood vessels on the surface of the cortex, a "tandem-lens" arrangement (macroscope) was used (Ratzlaff and Grinvald, 1991). This device is essentially a microscope, with a rather low magnification, composed of two back-to-back photographic lenses. The macroscope provides an unusually high numerical aperture compared to a commercial, low-magnification microscope objective. Consequently, this optical system has a very shallow depth of field (50 $\mu \mathrm{m}$, nominal). Therefore, when focused $300 \mu \mathrm{m}$ below the cortical surface, the surface vasculature is sufficiently blurred and artifacts from the surface vasculature virtually disappear. Figure 2 demonstrates the benefits of using the macroscope. This figure provides a comparison between two activity maps, one obtained with a conventional macro lens $(A)$ and the other with the aid of our homemade macroscope $(B)$.

Visual stimuli. We employed a visual stimulator based on an IBM $\mathrm{PC} / \mathrm{AT}$ equipped with an SGT + video-graphics board (Number Nine
Corporation, Lexington, MA). The software for this stimulator was developed by Kaare Christian, The Rockefeller University. The stimuli were displayed on a CRT screen in $60 \mathrm{~Hz}$ noninterlaced mode. To stimulate a large visual field the monitor was positioned at a distance of $30-45 \mathrm{~cm}$ from the animal so that it stimulated an angle of $40-60^{\circ}$ in the visual field, contralateral to the hemisphere investigated. The cats were stimulated binocularly with high-contrast square-wave gratings with a spatial frequency of 0.15 cycles/degree and a drift velocity of $15 \% \mathrm{sec}$

Data acquisition. For functional imaging we normally presented a particular stimulus to both eyes of the cat for $2 \mathrm{sec}$ and recorded seven frames of $450 \mathrm{msec}$ duration with the CCD camera. To allow the relaxation of activity-dependent vascular changes, this period of data acquisition was followed by an $8 \mathrm{sec}$ interstimulus interval. To minimize the nonselective activation of cortical regions by turning on the visual stimuli or by changing their orientation abruptly, a standing grating of the orientation that was to be presented in the next stimulus condition was displayed during the interstimulus interval. Subsequently, the grating started to drift, and images of the cortex were taken. To reduce the noise in the acquired images, signal averaging was used. To this end, each stimulus was presented 16-128 times, randomizing the order of appearance in order to average out any stimulus-dependent systematic errors such as adaptation or other plastic changes (Gilbert and Wiesel, 1990). In the best experiments, however, faint orientation maps could be already discerned in a single trial.

Optimization of the data acquisition protocol. It has been reported that the rise time of the intrinsic signal is $1-2 \mathrm{sec}$ while the latency for its onset is only 100-200 msec (Grinvald et al., 1986; Frostig et al., 1990). To optimize the signal-to-noise ratio in these experiments, we stimulated the cortex for $2 \mathrm{sec}$ only. A longer stimulus duration resulted in larger blood vessel artifacts in the optical maps, presumably originating from larger contributions of activity-dependent blood volume and blood flow changes. The duration of the data acquisition was approximately $3 \mathrm{sec}$. Figure 3 shows the development of a functional map 


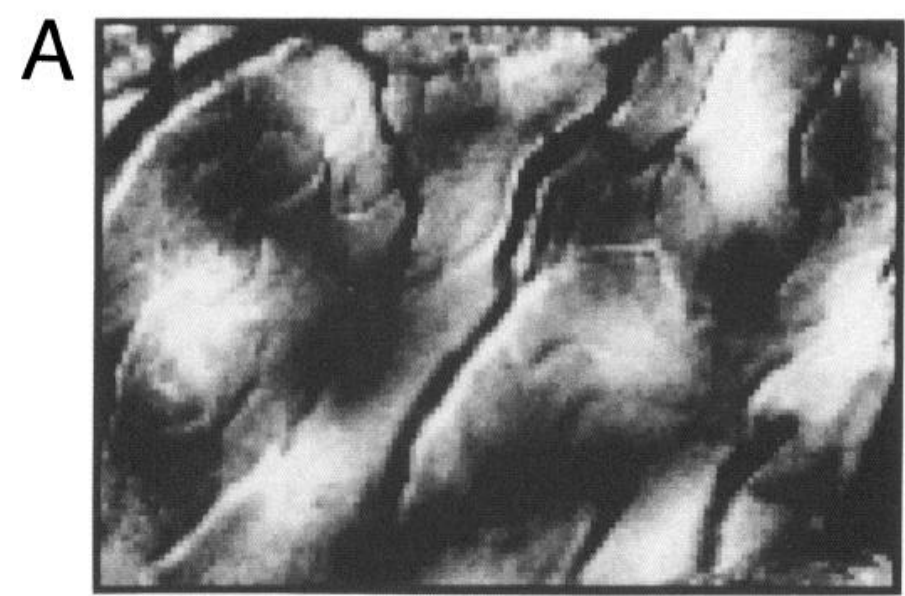

B

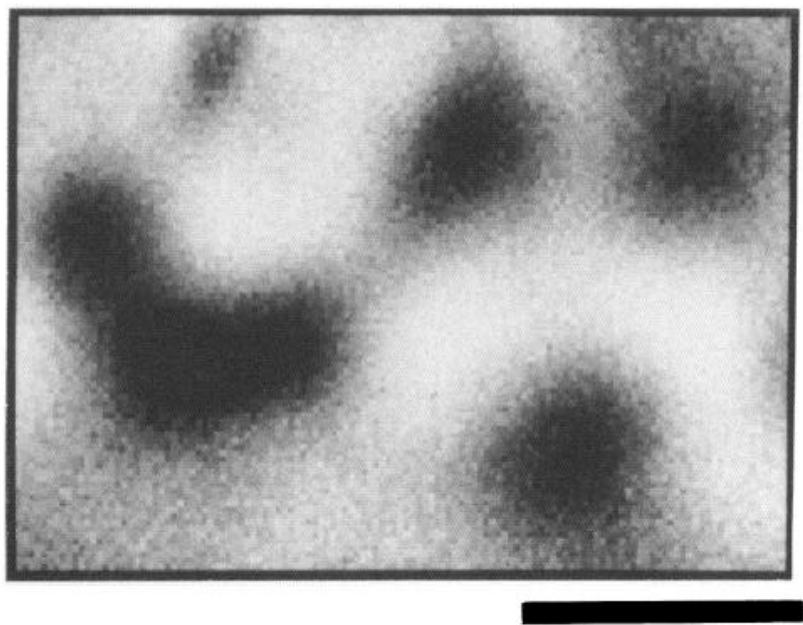

Figure 2. Improvements of functional imaging by the macroscope. $A$, An activity map obtained when the cortex was stimulated by a vertical grating. Cortical images were taken with a $100 \mathrm{~mm}$ macro lens coupled to a $2 \times$ extender. $B$, A similar map from another cat obtained with the macroscope. In both experiments the camera was focused $300 \mu$ m below the cortical surface, but the blood vessels artifacts (e.g., $A$ ) virtually disappeared only when the macroscope was used $(B)$. Scale bar, $1 \mathrm{~mm}$.

as a function of time. To illustrate this, four of the seven frames acquired are shown with the same scaling. One can see how the optically recorded map improves with time. Since the signal-to-noise ratio of frames obtained at short latency was already acceptable, they were also included in the present analysis. We repeatedly observed that functional maps could be observed also from standing rather than drifting gratings. While this observation was not explored further, it clearly contributed to the intensity of the map observed at the onset of the drift (Fig. $3 A$ ).

Data analysis. The first step in the data analysis was to integrate over the frames acquired for each visual stimulus (see Fig. 3). To remove the baseline image and to correct for the effects of uneven illumination and other common mode noise from the microvasculature, we divided each of these summed images by a "blank image." 2 The resulting maps are referred to as "single-condition maps." The blank image was obtained by two different procedures. In some experiments the pictures for all different stimuli were added together and are referred to as a "cocktail blank." In others we used the frames acquired during a trial in which the cat viewed a blank screen ("genuine blank"). In most cases the cocktail blank was used since it provides an image of activated cortex, whereas the genuine blank is taken from an unactivated brain.

${ }^{2}$ Division is mathematically equivalent to subtraction of the two images after their correction for uneven illumination (D. Shoham, personal communication).
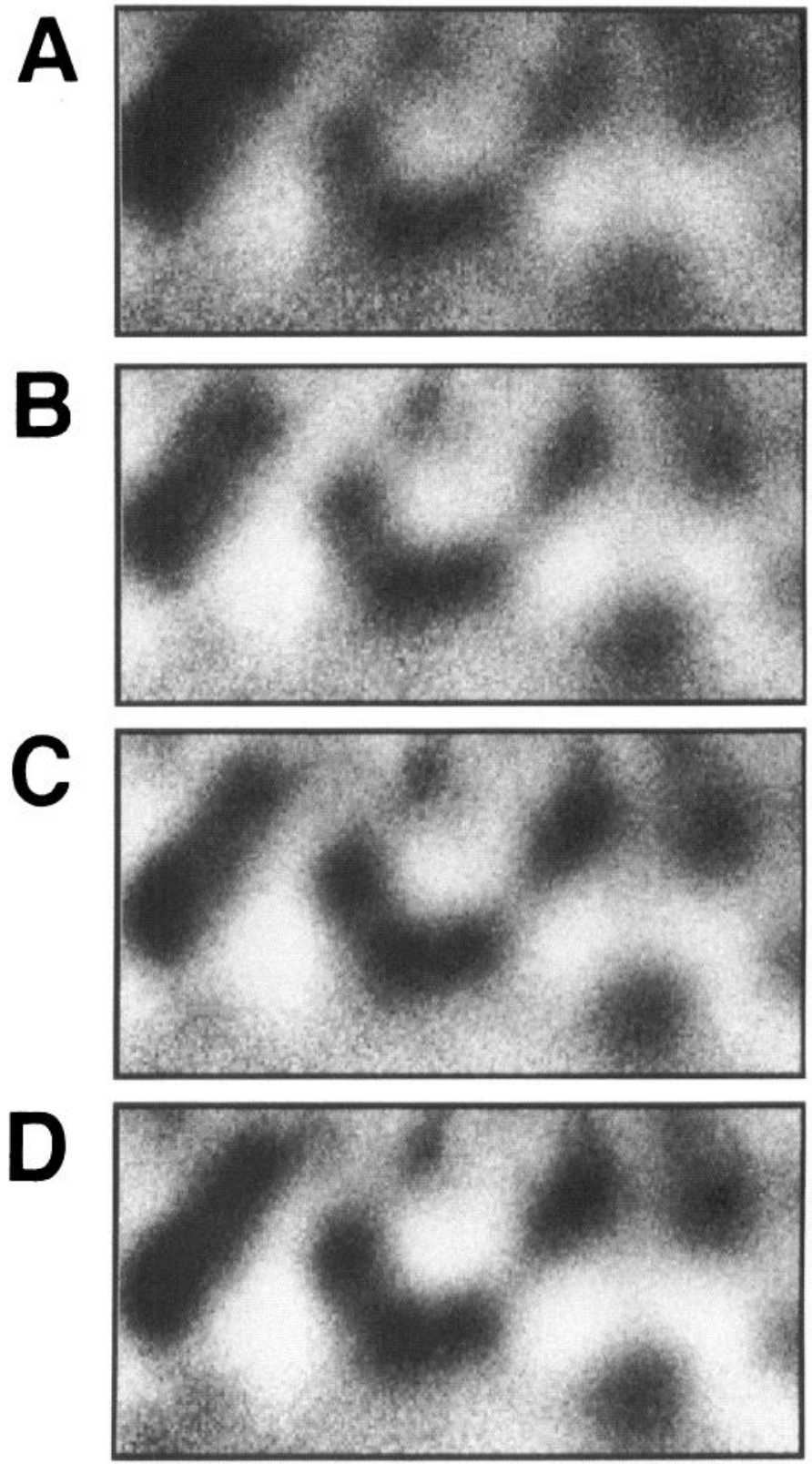

Figure 3. Development of functional maps as a function of time: four of seven consecutive frames showing functional maps. Each frame lasted $450 \mathrm{msec}$. The first frame started at the onset of the visual stimulus, yet a clear functional map can already be observed. The other three frames were taken starting at 900,1600 , and $2400 \mathrm{msec}$, respectively, after the stimulus onset. Scale bar, $1 \mathrm{~mm}$.

Therefore, usually much larger blood vessel artifacts were observed when the genuine blank picture was used. On the other hand, it is valid to use a cocktail blank only as long as a control map obtained by dividing the cocktail blank by the genuine blank provided a flat uniform map relative to the functional map under investigation. Only vascular artifacts should be apparent; otherwise, the cocktail blank may distort the resulting functional images. It should be noted here that none of the functional maps presented in this study were smoothed. We used the 2 $\times 2$ pixel binning described above, without any reexpansion to apparent higher resolution.

For comprehensive analysis of the organization of iso-orientation domains, the responses for eight different gratings were summed vectorially on a pixel-by-pixel basis (Blasdel and Salama, 1986; Ts'o et al., 
1990). For every point in the cortex we summed eight vectors, their lengths being the magnitude of the "single-condition responses" and their angles corresponding to the orientation of the gratings that produced the responses. (To map the $180^{\circ}$ onto a full $360^{\circ}$ circle, the angles of the different orientations were first multiplied by two.) There are several ways to display the results of the vectorial analysis, each of which emphasizes a particular aspect of the organization of iso-orientation domains. One approach is to display only the angle of the resulting vector (having divided it by two) and produce an "angle map": the colors from yellow through green, blue, red, and back to yellow are codes for the angle of the preferred grating for this piece of cortex. Alternatively, additional information may be provided; the magnitude of the resulting vector can be also displayed as the brightness (i.e., intensity) of the color. The resulting "polar" map (termed "angle-magnitude map" in Bartfeld and Grinvald, 1992) then shows the preferred orientation (hue of the color) and the magnitude of the vector (intensity of the color) at the same time. Note, however, that a vector with a low magnitude can be the result either of various stimulus orientations evoking the same strong response, or simply of a weak response to all orientations.

Fracture analysis. To analyze the rate of orientation change, we applied a two-dimensional gradient operator to the orientation preference maps. This was carried out taking the circular nature of orientation data into account; that is to say, the difference between a preferred angle of $170^{\circ}$ and $10^{\circ}$ was defined as being $20^{\circ}$ and not $160^{\circ}$. The formula that we used to calculate the gradient is

$$
\begin{aligned}
& |\nabla I(x, y)| \\
& =\sqrt{\left[\frac{\partial I(x, y)}{\partial x}\right]^{2}+\left[\frac{\partial I(x, y)}{\partial y}\right]^{2}} \\
& \approx \sqrt{[I(x+1, y)-I(x-1, y)]^{2}+[I(x, y+1)-I(x, y-1)]^{2}},
\end{aligned}
$$

with $I(x, y)$ being the "angle map" and $x, y$ the coordinates of the single pixels in the map.

$|\nabla I(x, y)|$ was then mapped onto a gray scale such that in the resulting image white areas coded for a high rate of orientation change and dark areas for slow orientation changes.

Reliability of optical maps. To demonstrate the reliability of the data, two examples of the reproducibility of the optical maps are presented here. Figure 4 illustrates the reproducibility of single-condition maps, by comparing functional maps obtained from two independent imaging sessions on the same patch of cortex. Similarly, Figure 5 illustrates the reproducibility of two independent angle maps, obtained from the same piece of data. This high degree of reproducibility gives us confidence in the quality of our data and facilitated the detailed evaluation of the functional architecture in cat visual cortex.

\section{Results}

\section{Mapping of iso-orientation domains}

To obtain an iso-orientation map, we divided the cortical image taken when the animal viewed a grating of one orientation by the "cocktail blank" image. Figure $6, A$ and $B$, shows such singlecondition maps for two orthogonal orientations $\left(45^{\circ}\right.$ and $\left.135^{\circ}\right)$ taken from a cortical patch of $7 \times 4 \mathrm{~mm}$. Figure $6 C$ shows a "differential orientation map" (Blasdel, 1992a,b). In this picture the two activity maps were divided by each other. To show the relation of the functional maps to the image of the cortical region, an image of the cortical surface is shown in Figure $6 D$. To emphasize the vascular pattern, this image was taken when the cortex was illuminated with green light of $540 \mathrm{~nm}$ wavelength. The functional maps, on the other hand, were obtained using an orange light at a wavelength of $605 \mathrm{~nm} \mathrm{(20} \mathrm{nm} \mathrm{band-}$ width), an illumination wavelength under which the vascular pattern is much less visible.

There is a fundamental difference between "single-condition" and "differential" maps. In analyzing single-condition maps, one makes minimal assumptions about the underlying func-

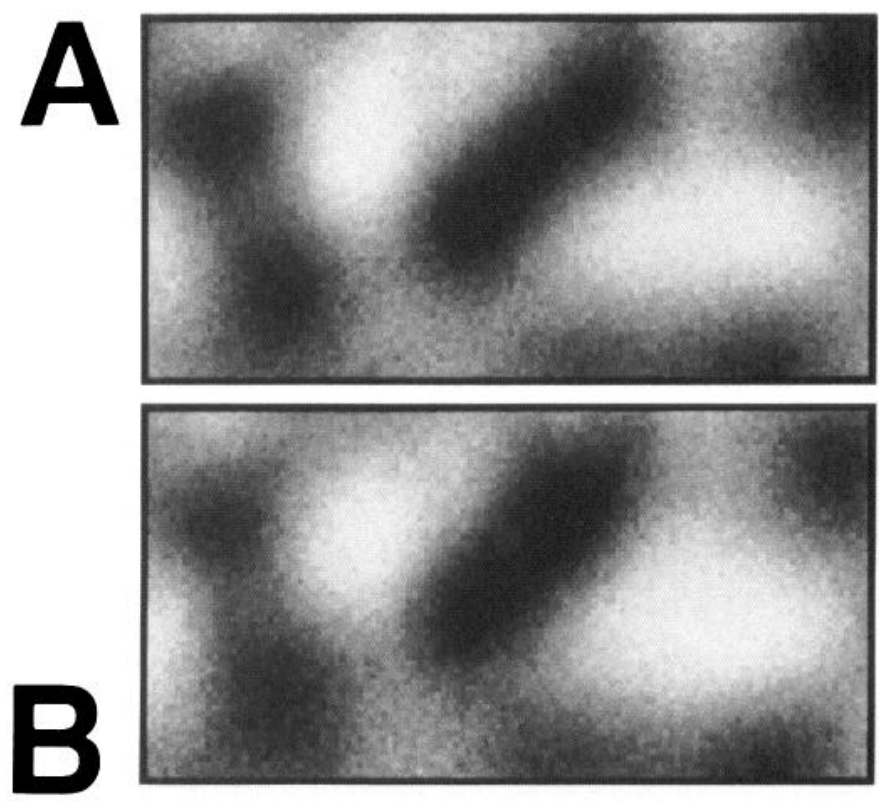

Figure 4. Reproducibility of iso-orientation maps. Two iso-orientation maps obtained in two independent imaging sessions from the same patch of cortex are shown. The experiment lasted $6 \mathrm{hr}$. $A$ shows a singlecondition map from the data acquired during the first, third, and fifth hours. $B$ shows the same single-condition map from second, fourth, and sixth hours. To determine the reproducibility of these maps they were also analytically compared. The RMS of the difference between the two images was only $6.5 \%$ of the full scale of gray values. For every one of these maps 48 responses were averaged. Scale bar, $1 \mathrm{~mm}$.

tional architecture. The image of the activated cortex has only been divided by the "blank image" (see Materials and Methods). Apart from this operation, this method provides an unprocessed activity map for one particular stimulus. In contrast, a differential map is obtained by dividing the activity map for one stimulus by the activity map for another. Although a significant contrast enhancement can be achieved, this procedure results in a loss of information, unless the two stimuli activate complementary parts of the imaged cortical area, which is often not the case. Thus, differential images provide higher contrast at the cost of masking the common mode response for the two stimuli. Therefore, we prefer to work with single-condition maps, thus minimizing the underlying assumptions.

\section{Directionality columns}

To determine whether cells are clustered into direction selective domains, two gratings of the same orientation, but moving in opposite directions, were used as stimuli. We compared the two activity maps to determine whether the direction of motion affected the resulting activity pattern. Figure $7 A$ shows the activity map for a vertical grating moving to the left. The map for the same grating moving to the right is shown in Figure $7 B$. Visual inspection of these two images suggests that the two maps are similar. Indeed, subtraction of these two maps results in the apparently patternless picture shown in Figure $7 C$ (using the same scaling as in $A$ and $B$ ). We analyzed orientation maps obtained from nine cats and found that maps obtained for a grating moving in one direction were similar to those obtained with a grating moving in the opposite direction. From these data 

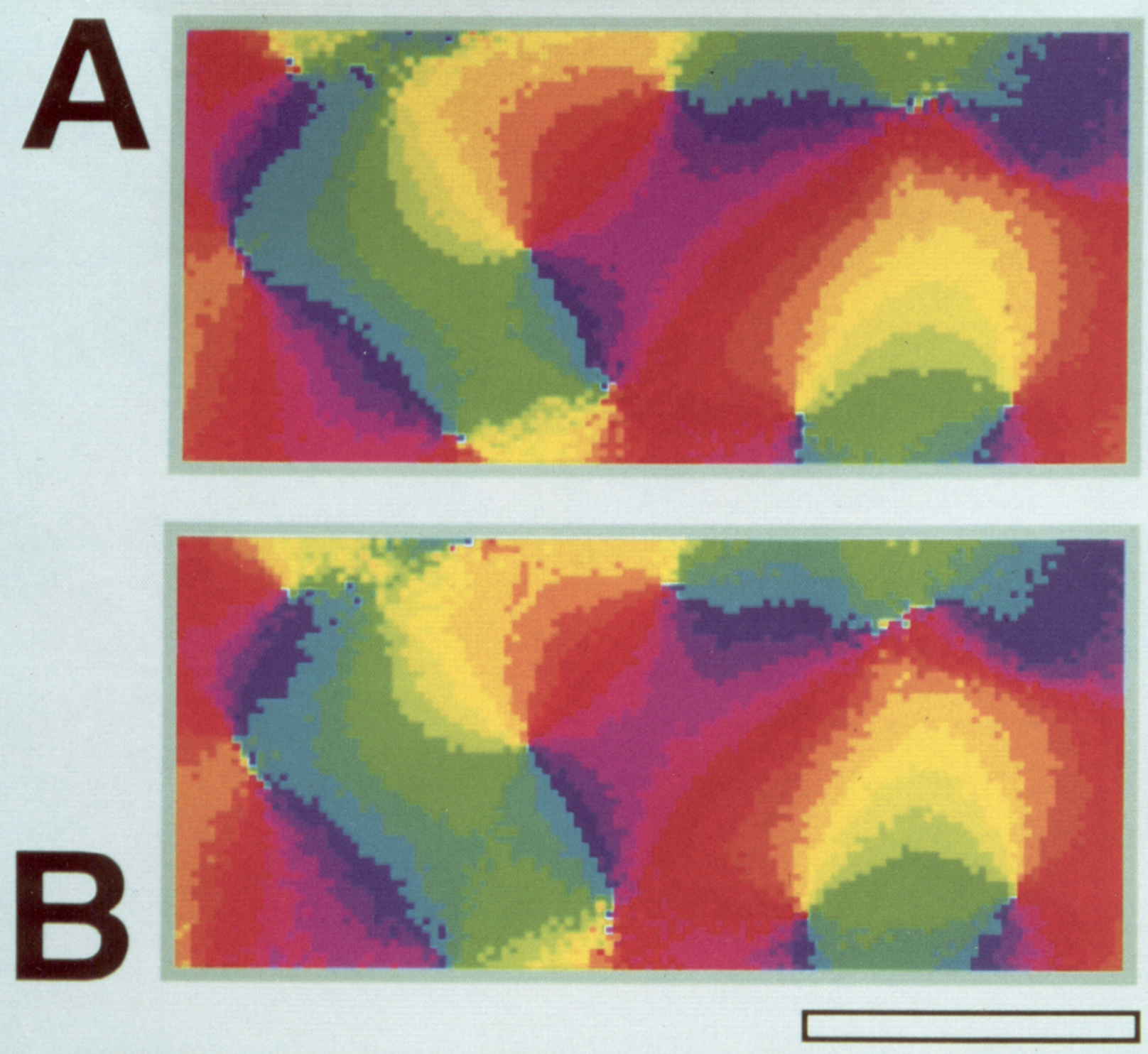

Figure 5. Reproducibility of angle maps. Two angle maps obtained in two independent imaging sessions from the same patch of cortex are shown. The experiment lasted $6 \mathrm{hr}$. $A$ shows an angle map from the data acquired during the first, third, and fifth hours. $B$ shows the same angle map from the second, fourth, and sixth hours. The RMS for the differences between the optimal angles detected at each pixel was only $9.8^{\circ}$, and for $88 \%$ of the pixels the deviation was less than $10^{\circ}$. The small areas of the map exhibiting reduced reproducibility between the two imaging sessions always corresponded to cortical regions with poor selectivity for the set of stimuli used. Scale bar, $1 \mathrm{~mm}$.

we conclude that cells in the upper layers of area 18 are not strongly clustered into directionality columns.

\section{The layout of iso-orientation domains}

To study the architecture of iso-orientation domains in detail we took advantage of the fact that optical imaging allows mapping of the responses to gratings of many orientations in one cortical region in the same animal. Figure 8 shows four isoorientation maps obtained by using stimuli of different orientations. Black regions again denote domains that are activated by the respective stimulus, while white regions are the minimally activated areas. Every single one of these maps shows the regions of the observed part of cortex that are metabolically active in response to a particular stimulus. It is therefore not surprising that these optical maps are similar to 2-DG maps. One prom- inent feature seen here is dark patches reminiscent of the "beads" (Löwel et al., 1987) that appear within the bands. However, upon careful comparison of the four maps, one can observe that areas that appear gray, connecting regions between two dark patches in one orientation, often correspond to dark beads in another, approximately $45^{\circ}$ different orientation. Our results, therefore, call for a reinterpretation of the 2-DG data from which it had been concluded that iso-orientation domains are bands with a beaded appearance.

Although iso-orientation domains are definitely not elongated bands, we observed that many of the iso-orientation patches (Fig. 8) are asymmetric, slightly elongated in the mediolateral axis. This impression was corroborated by mathematical analyses, calculating autocorrelation functions for the iso-orientation maps. These 2-dimensional autocorrelation functions show 
A
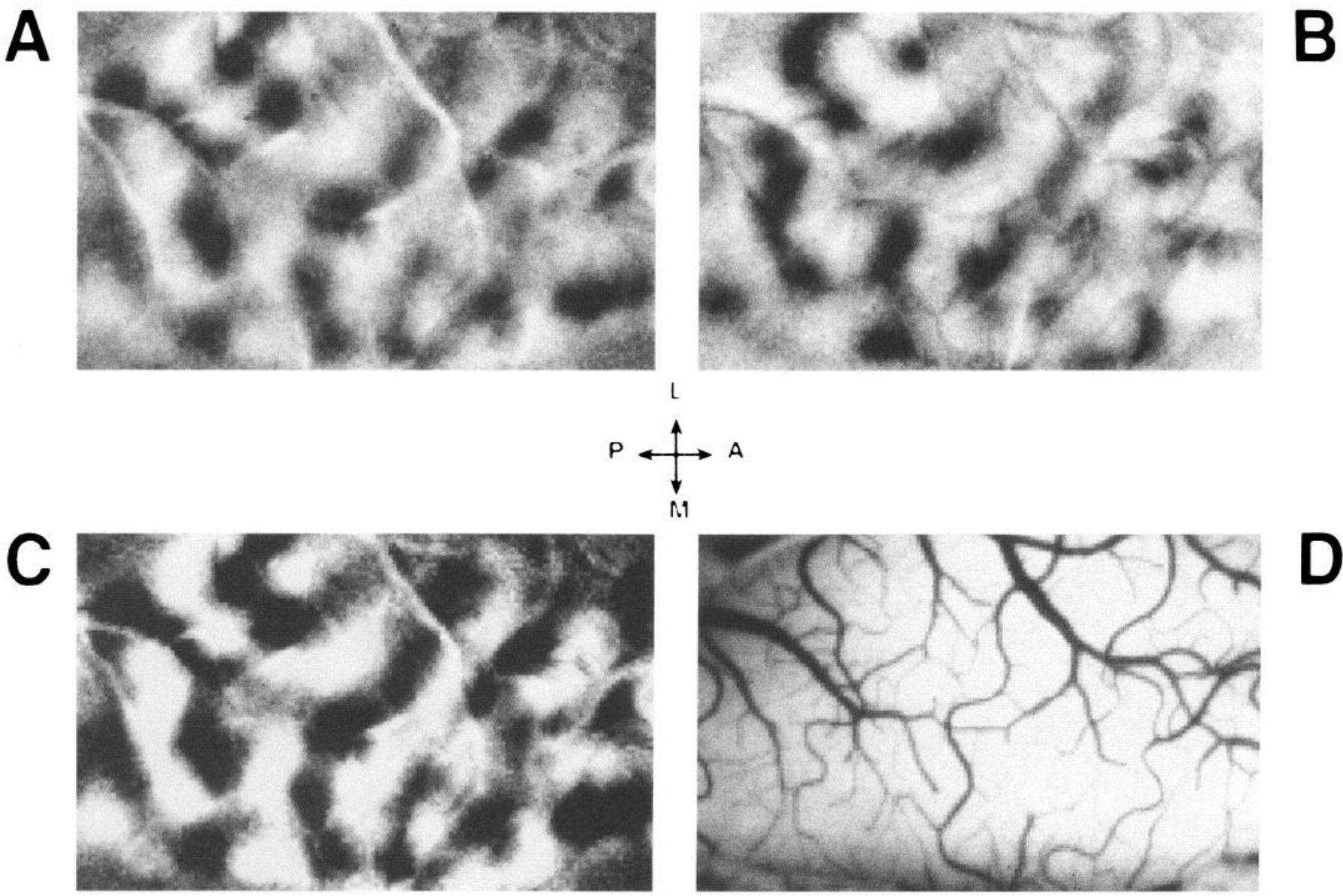

Figure 6. Single-condition iso-orientation maps from area 18 of cat visual cortex. $A$, Image acquired during stimulation of the cat with an oblique $\left(45^{\circ}\right)$ moving grating. To correct the effect of uneven illumination of the cortex and nonspecific vascular responses, the image was divided by the sum of the pictures obtained for all the different orientations. Dark areas in the image are regions of stronger light absorption and hence regions of strongest activity. $B$, Similar to the map in $A$, but this map was obtained with a stimulus of orthogonal orientation to that used in $A$. The resulting functional map is nearly the inverse of the map shown in $A$, indicating that regions strongly activated by one stimulus are only weakly activated by the orthogonal one and vice versa. $C$, Significant contrast enhancement can be achieved by dividing the image obtained with one stimulus $(A)$ by the image obtained with the orthogonal stimulus $(B)$. The images were acquired with the macro lens and not with the macroscope. The same scaling was used for the amplitude of the responses in $A-C$. D shows the blood vessel pattern of the imaged cortical area $(7 \times 5.6 \mathrm{~mm})$.

that the patches are asymmetric and that their axis of elongation usually lies in a mediotemporal (sometimes slightly anteriomedial/posteriotemporal) direction (D.-S. Kim and T. Bonhoeffer, personal communication).

\section{Radial arrangements and continuity in orientation preference maps}

To determine the overall organization of orientation preferences in a given cortical region, we combined the information from many activity maps using vectorial addition (Blasdel and Salama, 1986; Ts'o et al., 1990). Thus, from iso-orientation maps displayed in Figure 8, we calculated the preferred orientation on a pixel-by-pixel basis. The responses to the different orientations were summed vectorially (see Materials and Methods). In the resulting map (Fig. $9 B$ ) the colors code for the optimal stimulus orientation for each cortical site. The salient features of these maps are radial (sometimes slightly elongated) structures around points of singularity referred to as "orientation centers" (arrowheads in Fig. 9B). The various orientation preferences are grouped around these orientation centers in a continuous pinwheel-like fashion. In the following, with the word "pinwheel" we will refer to a region of approximately $1 \times 1$ $\mathrm{mm}^{2}$ containing a full set of radially arranged iso-orientation domains. The "pinwheel center" will be referred to as "orientation center." Figure 10 shows an enlargement of one of these pinwheels. Every orientation is represented once around the orientation center. This was found in all of the 147 pinwheels inspected in this study. The full sequence of orientations was never represented more than once around a center singularity. Additional examples can be seen in Figure 11, in which eight pinwheels, taken from maps recorded from different animals, are shown. This figure also illustrates another important aspect of these radial structures. There are two different categories of pinwheels, a clockwise and a counterclockwise one. The eight pinwheels in Figure 11 are divided into these two categories. The upper row shows pinwheels in which the preferred orientations change in a clockwise direction. In the lower row the orientations change in a counterclockwise fashion.

The sharp breaks between the different colors in the pinwheel in Figure 10 might give the impression that there are actually very well circumscribed domains that represent neurons of particular orientation preferences. This is not the case. We deliberately chose only 16 discrete colors for the whole range of orientations since the breaks between the colors emphasize the 

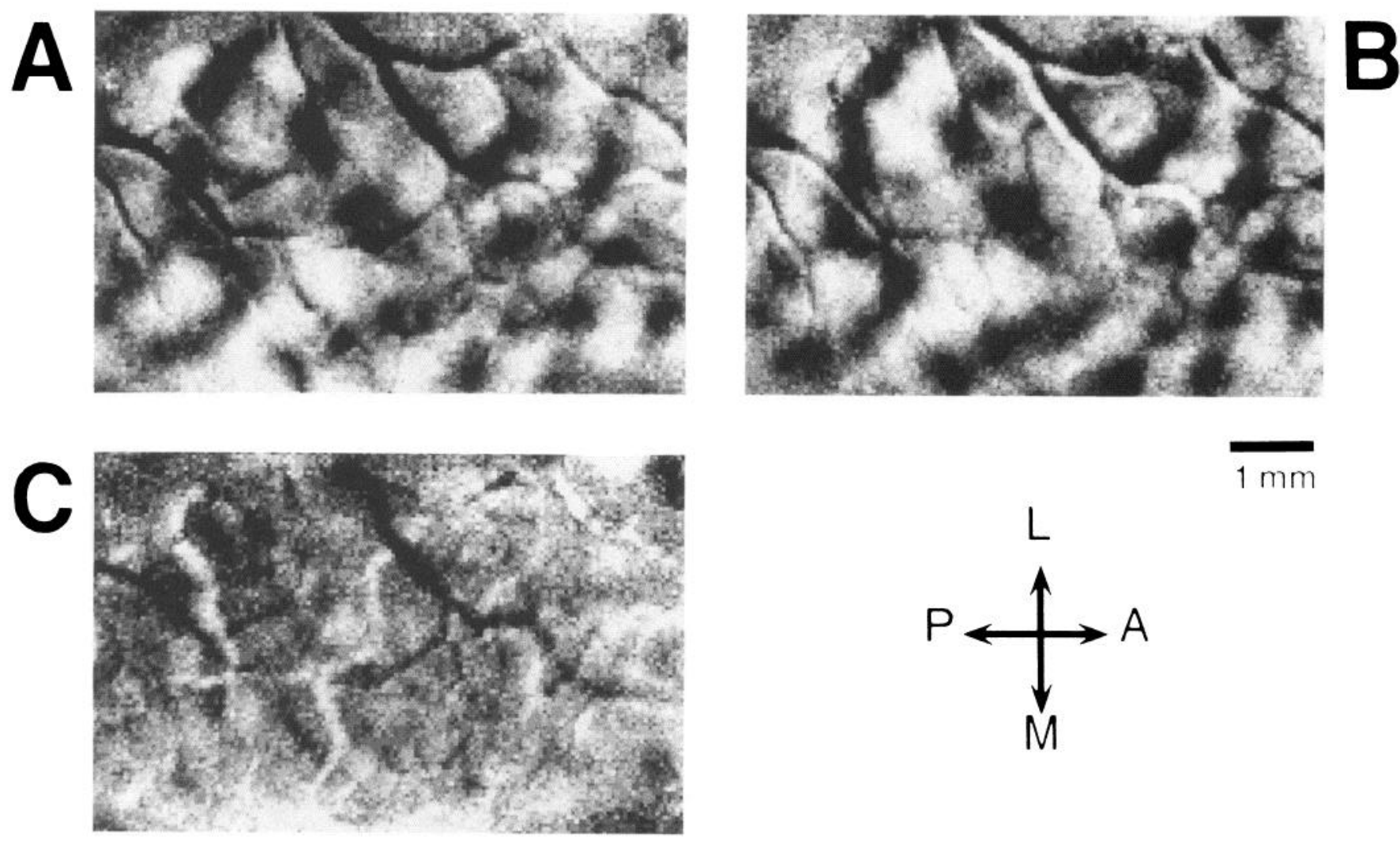

Figure 7. Lack of robust direction-selective domains in cat area 18. Mapping of direction-selective clusters is obtained with two gratings of the same orientation but moving in different directions. $A$, Iso-orientation single-condition map recorded with a vertical grating moving to the left. $B$, A similar map produced by the same vertical grating but moving to the right. $C, \mathrm{~A}$ nearly patternless picture is produced by subtracting these two images from each other. The images were obtained with the macro lens, not with the macroscope. Each stimulus was averaged 32 times. The same scaling was used for the amplitude of the responses in $A-C$.

radial nature of the pinwheels. Figure 12 now shows the same pinwheel as Figure 10, using a continuous color scale to code for preferred orientations. Although this image was not smoothed in any way, the preferred angle changes in a perfectly smooth and continuous fashion around the orientation center.

We found that counterclockwise (ccw) and clockwise (cw) pinwheels appear in approximately equal numbers $\left(n_{\mathrm{cw}}=80\right.$ and $n_{\text {ccw }}=67$ in the experiments that we analyzed in this respect). In fact, an analysis of how pinwheels are distributed in the cortex shows that often two adjacent pinwheels of opposite chirality are smoothly connected to each other such that thin "iso-orientation bands" are continuous between the two pinwheel centers. Figure 13 illustrates a typical example of two adjacent pinwheels.

\section{Orientation tuning curves for a population of neurons}

To compare the optical imaging data to data that are available from microelectrode recordings, we also computed orientation tuning curves directly from the optical data. This is shown in Figure 14 for 10 selected points. For every cortical site (i.e., every pixel), we determined the magnitude of the response to a grating of a certain orientation and plotted it as a bar, the color of the bar coding for the orientation of the stimulus. This process was repeated for all eight orientations and yielded a tuning curve for every point in the imaged area. Ten such tuning curves are displayed around the center map in Figure 14. The tuning curve in the upper left corner, for instance, was calculated for the point to which it is connected by the black line. The tuning curves generally look similar to the ones obtained from conventional electrophysiological recordings, except that they appear to be somewhat broader.

We were particularly interested in examining the orientation tuning curves in the orientation centers. Two such tuning curves are shown directly above and directly below the orientation map in Figure 14. Both of these curves are rather flat when compared with the tuning curves that were obtained in sites remote from the orientation centers.

\section{"Tangential penetrations" through orientation maps}

To determine how the optical imaging data compare with previous electrical recordings, we calculated how orientation preference changed with distance along a particular track, through the orientation preference map (angle map). Figure 15 shows two such "tangential penetrations" obtained from the optical data. One is where the sequence of orientations changes gradually, initially in a clockwise direction and later in a counterclockwise direction. The second track shows a different example where the orientation preference changes gradually at first and then suddenly a jump of $\sim 90^{\circ}$ occurs. Both curves obtained from the optical data are almost indistinguishable from the results observed in long tangential electrode penetrations (Hubel and Wiesel, 1974; Albus, 1975, 1985). These examples, then, show that the optical imaging data are fully consistent with data gathered using classical techniques. 

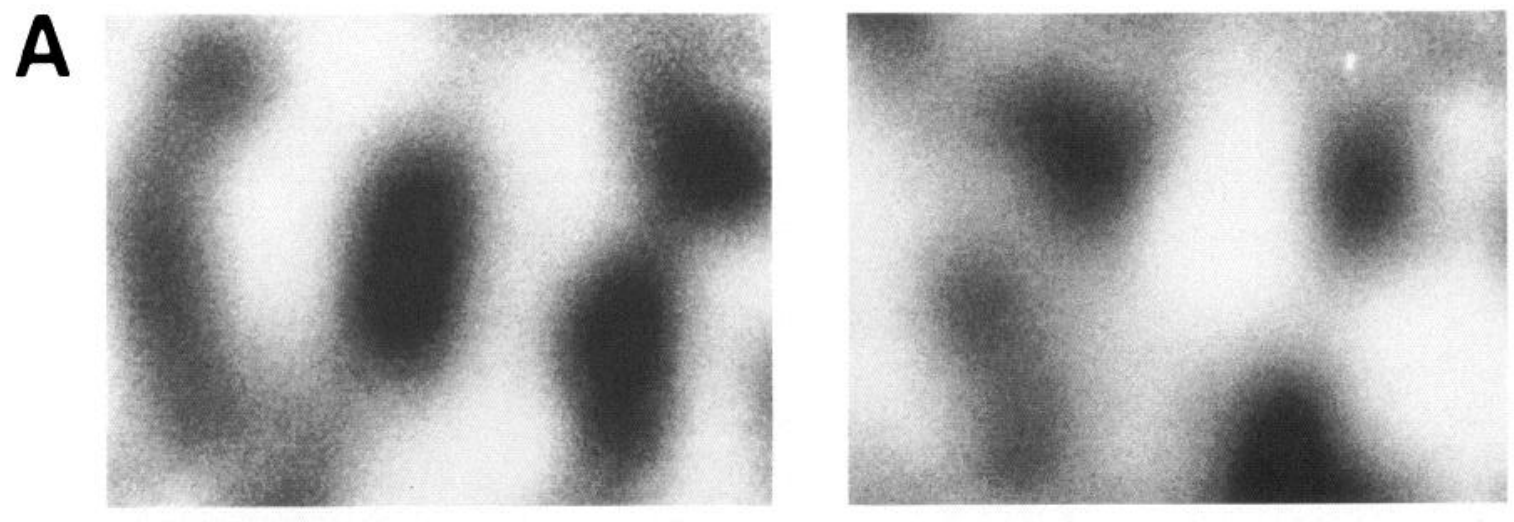

B
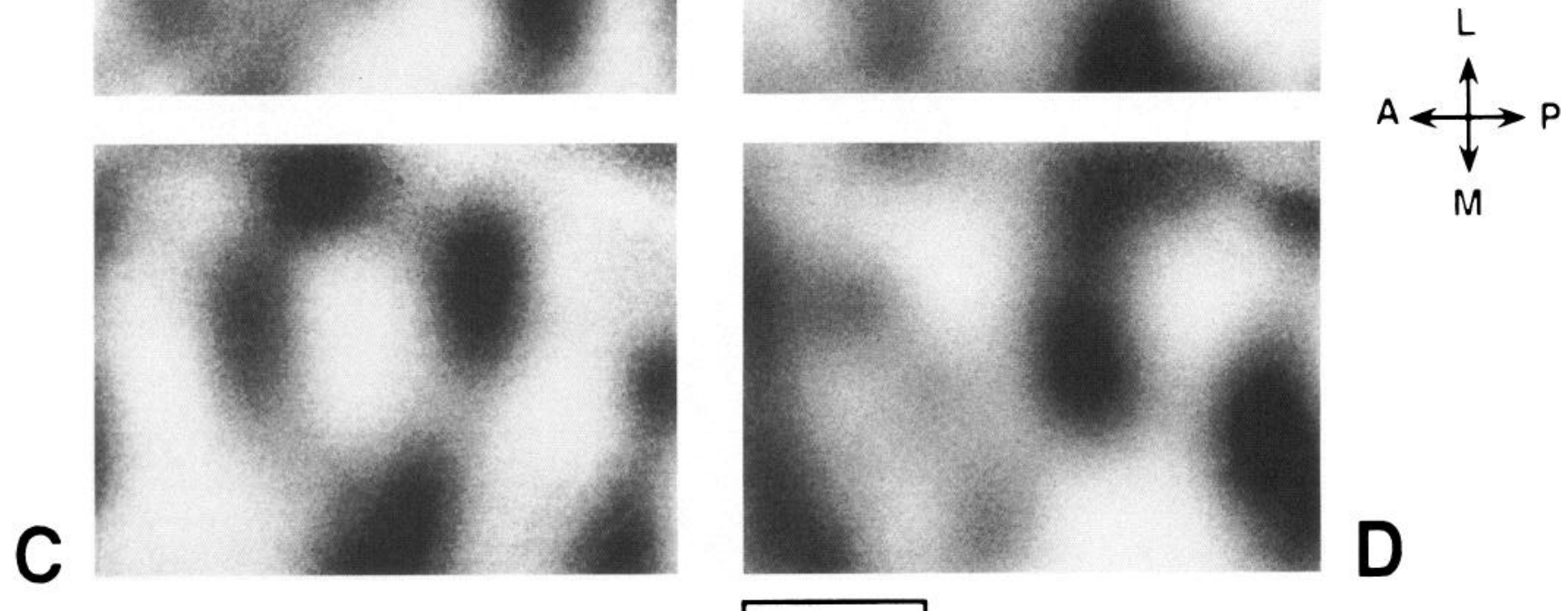

Figure 8. Beaded appearance of iso-orientation maps. A set of four iso-orientation single-condition maps was obtained by moving gratings of different orientations $\left(A, 0^{\circ} ; B, 45^{\circ} ; C, 90^{\circ} ; D, 135^{\circ}\right)$. A characteristic feature of all four maps is their beaded appearance: dark regions of very strong activation are connected by lighter regions of weaker activation. Each stimulus was averaged 48 times. Scale bar, $1 \mathrm{~mm}$.

\section{Polar maps}

To convey additional information present in the optical data, one can use a different method of displaying the data. From the vectorial addition that was used to calculate the angle map, we also obtained the magnitude of the resulting vector. The two parameters (preferred angle and magnitude of the averaged response resulting from vectorial addition) were then combined into one single picture. Figure 16 shows such a "polar map" in which the color codes for the angle of the preferred orientation whereas the brightness of any given point shows the magnitude of the resulting vector. This magnitude constitutes a measure of the sharpness in orientation tuning of the respective cortical area. For example, if a region showed equal responses for all orientations, the resulting magnitude of the vector would be zero.

In the polar map, shown in Figure 16, the pinwheels are again clearly visible. However, in this polar map, in contrast to the maps presented previously, one can also assess the quality of orientation tuning in different cortical loci. While most regions are very well oriented, orientation centers and their immediate vicinity show poor orientation tuning. This is in agreement with the data shown in Figure 14, where the orientation centers themselves show a very broad orientation tuning.

Single-unit recordings were used to distinguish between two possible interpretations of the poor orientation tuning at or near the singularity points depicted in Figures 14 and 16. (1) It is conceivable that all the neurons close to the orientation centers have broad tuning characteristics. (2) It is, however, also possible that single cells in the orientation centers are very well tuned, with the preferred orientation of the cells changing over very short distances. One pixel would then integrate responses from cells of many different preferred orientations and therefore the ensemble of cells, as a whole, would exhibit a broad orientation tuning. In a preliminary exploration, 12 such recordings, at or very near the singularity points, first imaged optically, showed that most neurons - even those very close to orientation centers-have a sharp orientation tuning. Although we could never be sure of exactly hitting the orientation center, it is likely that the broad orientation tuning in the orientation centers is brought about by a mixture of well-tuned cells with different preferred orientations.

\section{"Fractures"}

Hubel and Wiesel (1974) originally described how in most tangential penetrations changes from one orientation to the other were smooth. Preferred orientation changed gradually in clockwise or counterclockwise fashion, sometimes reversing from counterclockwise to clockwise and vice versa. In some instances, however, they also observed that orientation changes abruptly over very short distances. In many of the cases observed in monkey (Hubel and Wiesel, 1974) and in cat (Albus, 1975, 1985), these changes were jumps in orientation preference of usually $\sim 90^{\circ}$. Blasdel and Salama (1986) reported numerous locations of very fast orientation changes - the so-called "fractures"-in monkey V1 that could coincide with the electro- 


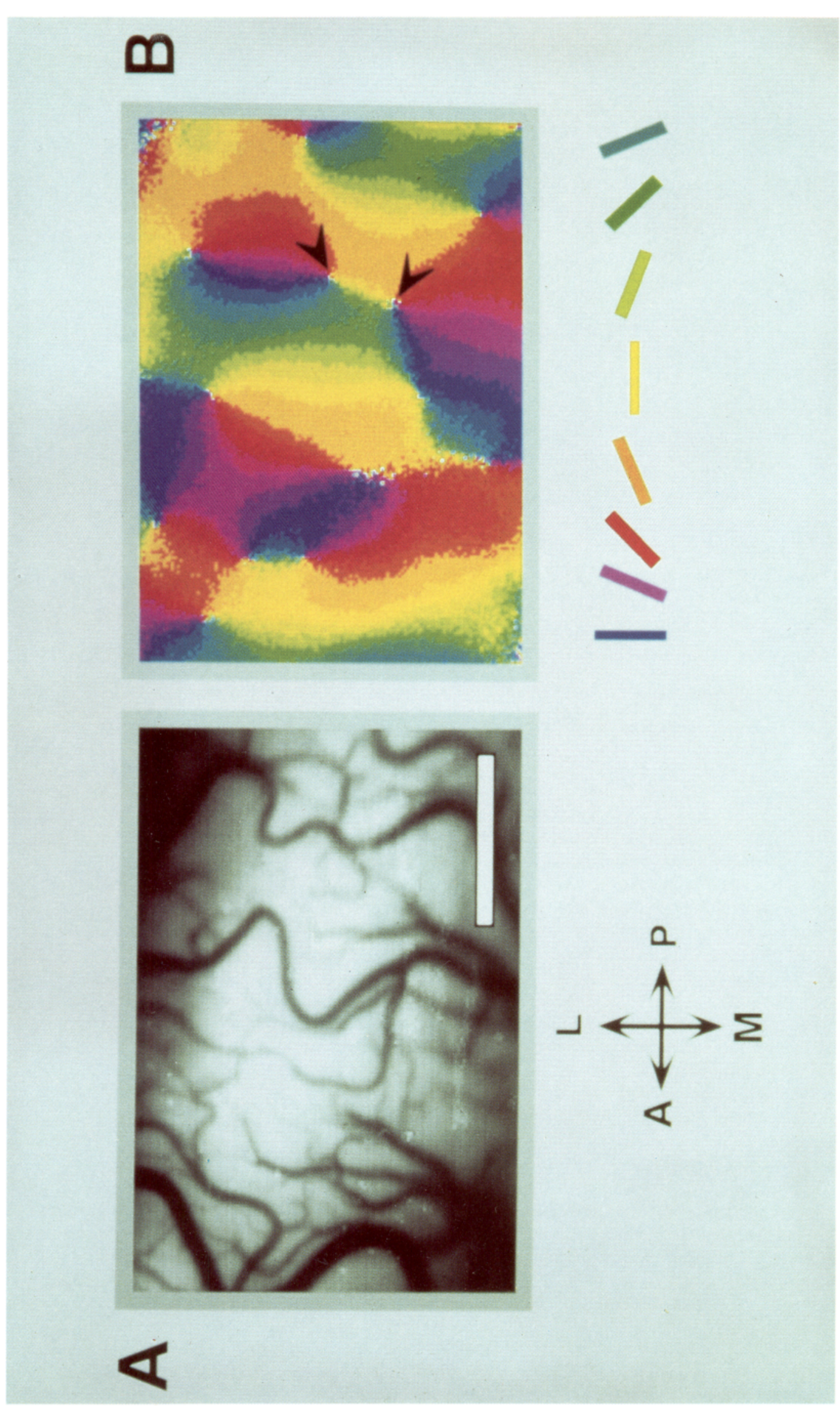

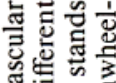
政 政

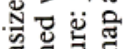
․ㅡㅇㅢ 틍으ㅇㅝㅗ 은 视节 일

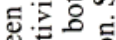

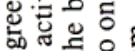
$\Xi \cong$ ธี

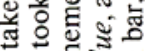

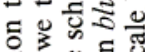
政 든 䠰

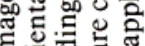
ㄷㅎㅇㅎㄴ ङ 엉요 으멸

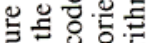
일. 헝 형 《记 ×혈

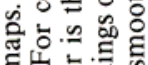
넌흐

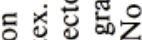
응 낭 苟吉壱 잉 ․ㅡㄴ. 도용 녕연 응 ठ․ㅠ 동요

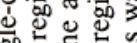
辰 क

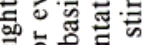
웡웜동 들 조. 을 空哥

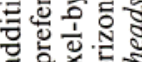

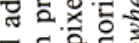

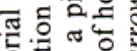

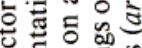
跣 ह 은 원 에 득 틍

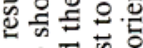

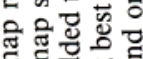
ह छ चृ

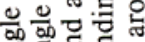
安完 등 任言总 远起

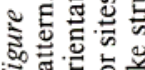




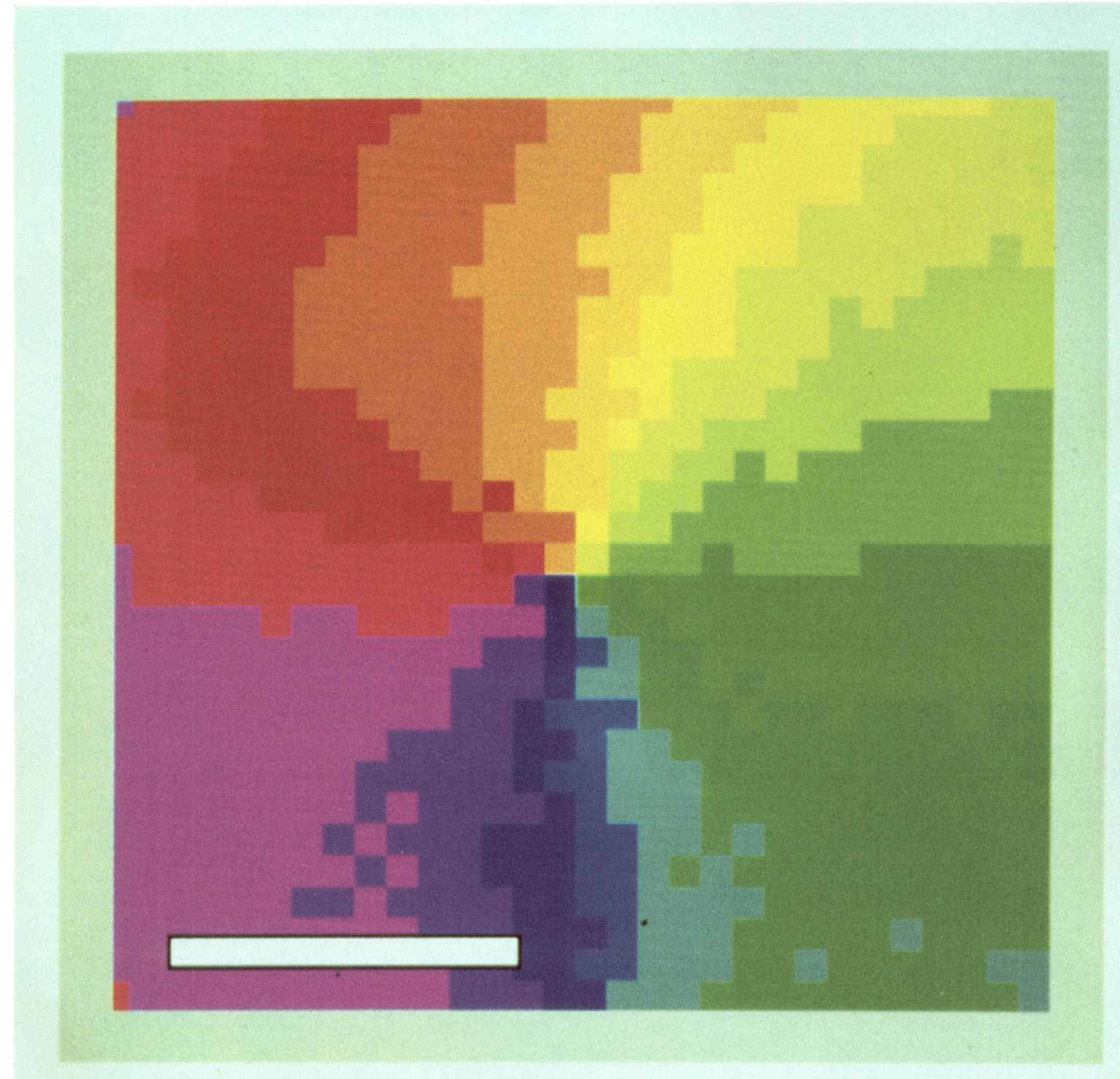

Figure 10. Close-up image of a pinwheel of different orientation preferences surrounding an orientation center. Note that every orientation appears only once around the center. The color coding is the same as in Figure 9. Scale bar, $100 \mu \mathrm{m}$.

physiologically observed regions of abrupt changes in orientation preference.

We examined whether in cat area 18 the jumps in orientation preference often seen in tangential electrode penetrations can be explained by the traversing of the microelectrode through a singularity point, or whether there are additional elongated discontinuities (fracture lines or fracture loops), along which preferred orientation changes very rapidly. To determine the rate of orientation preference change, a two-dimensional gradient operator was applied to the angle maps. We found points in which the rate of orientation change was very high. These points coincide with the orientation centers (where orientation changes are obviously strong). Apart from the orientation centers we did not observe any other cortical loci where orientation changed very rapidly. This can be seen in Figure $17 A$, which shows an example of a gradient map from area 18. In this gradient map white codes for strong changes in orientation preference. The white regions are always spots rather than elongated lines. Furthermore, these spots coincide with the orientation centers (compare the pattern with the map in Fig. 16). We found similar results in 21 orientation preference maps that were of sufficient quality to perform this kind of analysis. From this we conclude that in contrast to the numerous fractures reported for monkey V1 (Blasdel and Salama, 1986), the map of orientation preference in cat area 18 is smooth, except for the centers of the pinwheels.

\section{Are all orientations equally represented on the cortical surface?}

It is indeed a long-standing debate whether all orientations are equally represented on the cortical surface. Our data puts us in the fortunate position of having a very good sample (approximately 27,000 points per map) for resolving this question. We therefore reexamined our angle maps and plotted an orientation histogram of three animals from which we had maps of sufficient size and superior quality. The bar plot histogram in Figure 18 displays the relative areas devoted to processing of each particular orientation with a resolution of $22.5^{\circ}$. It can be seen that in the regions of area 18 , imaged in this study, the amount of 


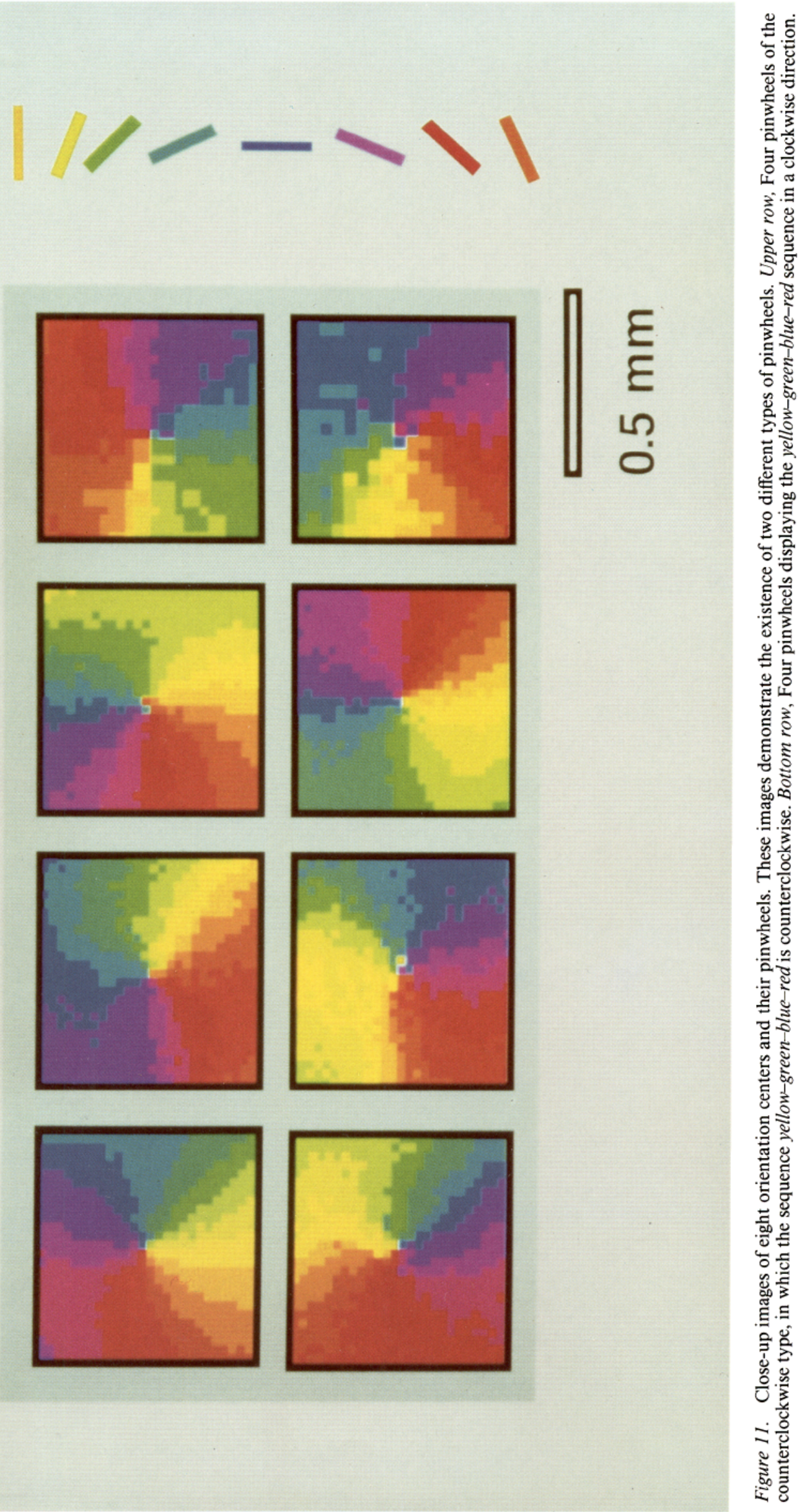




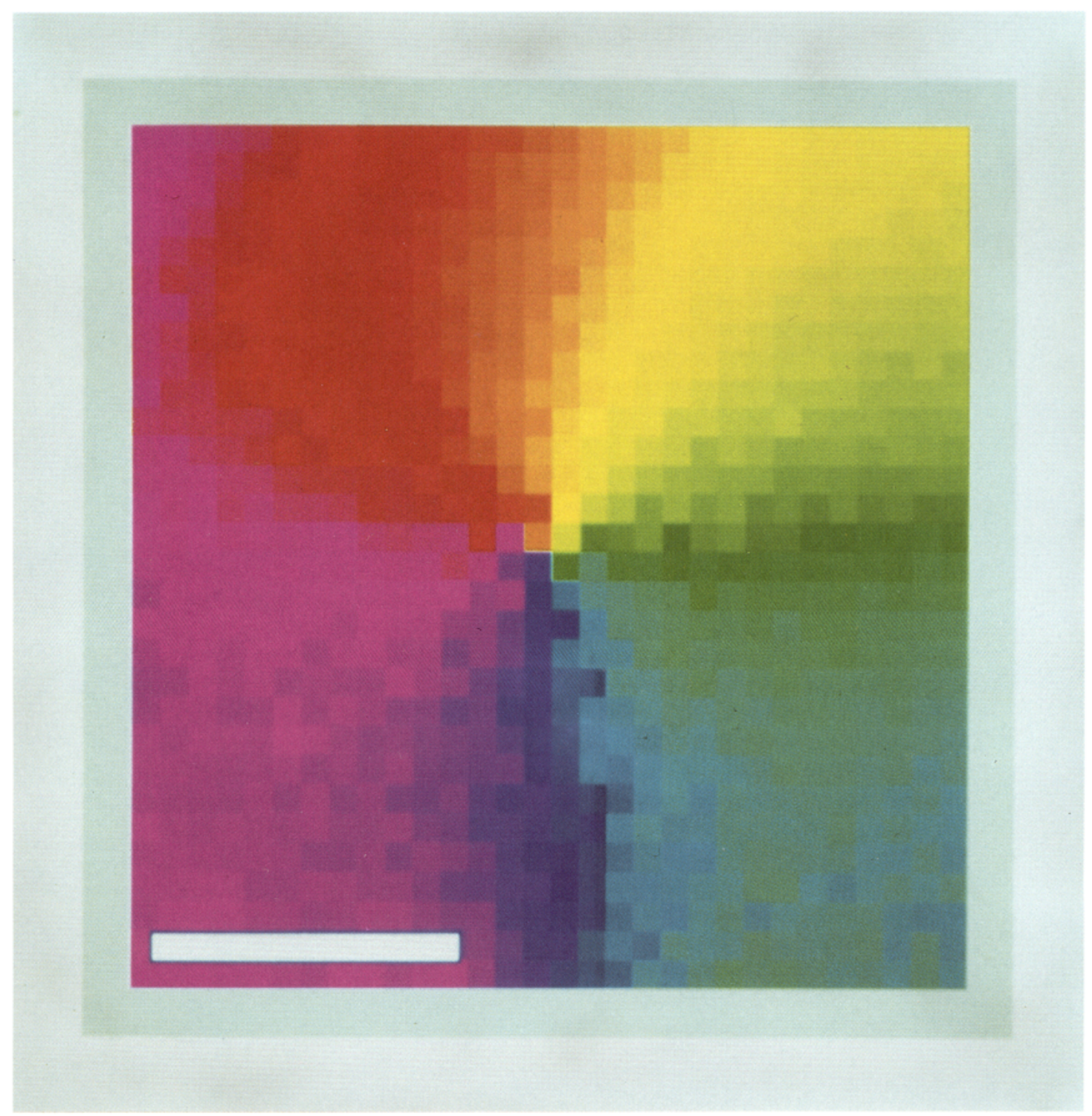

Figure 12. Continuity of iso-orientation domains. A pinwheel is shown using a continuous color scale. The apparent discontinuities introduced into Figure 10 by the use of only 16 colors vanish. Scale bar, $1 \mathrm{~mm}$.

cortex devoted to the processing of different orientations shows a uniform distribution.

We have shown that area 18 is parcelated according to the orientation preference of cells and not according to directionality. So far, we have not found further attributes of the visual stimulus according to which area 18 is organized. However, there are indications that such attributes exist: stimulation of the cortex with all orientations often activated the cortex in a patchy, nonuniform way (Fig. 19). This was observed in a number of experiments, raising the possibility that some neurons are clustered according to response properties remaining to be unveiled.

\section{Discussion}

The visual cortex of the cat has been extensively investigated by many groups using different techniques. Hubel and Wiesel (1962) found that cells with similar orientation preference are grouped together in space. However, the exact layout of isoorientation domains across the cortical surface and the principle underlying this organization have remained open issues. This can probably be attributed to particular limitations associated with the techniques uscd. Onc approach was to use multiple electrode recordings; the other, to utilize a radioactively labeled metabolic marker, 2-DG. Both techniques led to significant advances in the understanding of cortical maps but nevertheless suffer from significant limitations. In the past, 2-DG has only furnished information about the response to one particular stimulus (e.g., one particular orientation). Extrapolation of results obtained from such single maps to the overall organization of a cortical area is not always straightforward. The 2-DG technique, however, also offers some significant advantages over optical imaging; for example, rich information can be obtained from individual cortical layers at very high resolution and from deep brain structures (like the LGN) not at all accessible to optical imaging (e.g., Tootell et al., 1988). With multiple electrode penetrations one can obtain detailed information about different orientations but this method only provides a map with a limited spatial resolution. Optical imaging based on intrinsic 


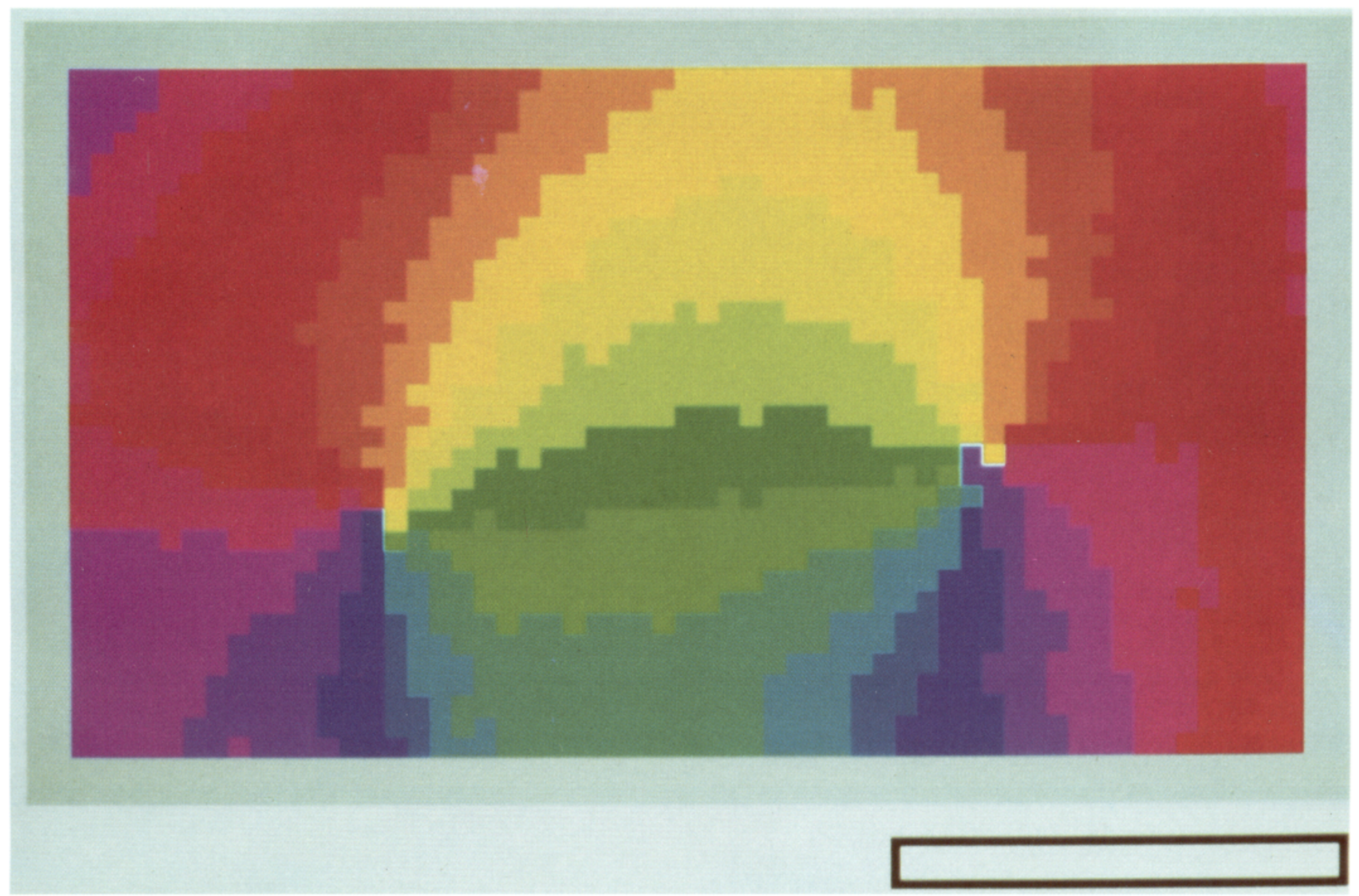

Figure 13. Continuity of iso-orientation domains between adjacent pinwheels: a close-up view of two adjacent pinwheels, one showing a clockwise orientation preference change, the other containing a counterclockwise sequence. Many adjacent pinwheels are smoothly connected to each other in this fashion. Thus, borders between neighboring pinwheels are not well defined. Scale bar, $500 \mu \mathrm{m}$.

signals, despite its own limitations, does not suffer from these two drawbacks and is therefore an ideal technique for assessing the functional organization of cortical areas lying on the surface.

\section{What does the optical signal represent?}

To interpret the results obtained by optical imaging based on intrinsic signals, it is important to remember that the optical maps provide information about metabolic rather than electrical activity. Although it is conceivable that these two types of activities are not related linearly to each other, a good correlation between electrical activity and the intrinsic optical signals has been obscrved (Grinvald et al., 1986). Furthermore, recent experiments on area MT of the owl monkey have shown an excellent correlation between optical and 2-DG functional maps (R. B. H. Tootell, D. Malonek, and A. Grinvald, personal communication).

The information obtained from optical imaging is related to population activity rather than to activity in single cells. The resolution of the technique is limited by the density of the microcapillary network, which is a mesh with an average spacing of $50 \mu \mathrm{m}$ (Scharrer, 1944; Kuffler et al., 1984). While the resolution across the cortical surface can be excellent, the depth resolution is not nearly as good. The optical maps probably average the vascular activity in the cortex down to a depth of 600-900 $\mu \mathrm{m}$ (Malonek et al., 1990), that is, the supragranular layers. At present we do not know if electrical activity of cell somata in the infragranular layers affects the optical maps. However, since many features of stimulus selectivity in the cortex are organized in a columnar fashion, the limited depth resolution does not severely hamper the imaging of cortical maps.

It is worth emphasizing that our optical imaging data provide information about average response properties of populations of neurons rather than single cells. In the majority of experiments each pixel viewed $23 \times 23 \mu \mathrm{m}^{2}$ of the upper part of a cortical "microcolumn" containing approximately 100 neurons from pia to white matter. It is partly for this reason that response properties assessed by optical recording are often not as well defined as one might expect from single-neuron recordings. For example, the orientation tuning curves in Figure 14 are rather broad. This can in part be explained by the jitter of preferred orientation and the different tuning curves that closely adjacent cortical neurons are known to have. In addition, the spatial resolution of optical imaging (discussed later) also affects the sharpness of response properties.

\section{Single-condition maps}

In the initial paragraph of the Results we emphasized that for optimal analysis of functional maps, it is essential to have highquality single-condition maps. To obtain such maps one takes the cortical image during presentation of one particular stim- 


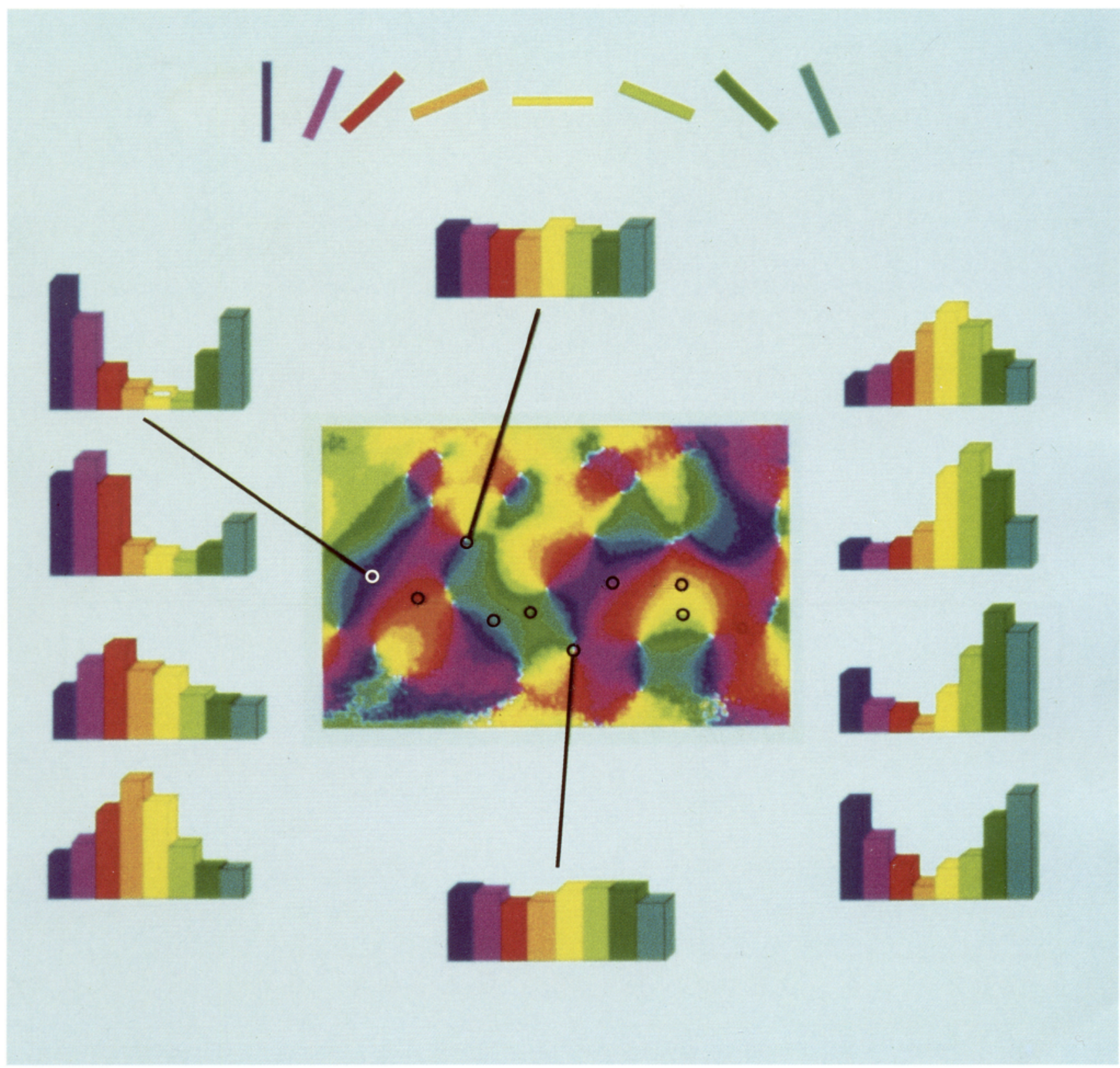

Figure 14. Orientation tuning curves for neuronal populations. The points for which the tuning curves (i.e., the bar plots) were calculated are marked with small circles. In every plot the magnitude of each bar indicates the strength of the optical response and the color codes for the orientation according to the scheme at the top of the figure. In most points the orientation tuning curves are similar to the ones that one would be obtained with conventional electrophysiology (see text). At the orientation centers, however, the orientation tuning profiles are flat (plots above and below the orientation preference map).

ulus, and corrects it only for the inevitable uneven illumination, dividing it by the blank picture. No further assumptions should be made. In previous reports using optical imaging, a different approach was taken: an image obtained with one stimulus was subtracted from the cortical image obtained with the orthogonal stimulus (Blasdel and Salama, 1986; Blasdel, 1992a,b). Although this procedure normally enhances the contrast of the pictures (e.g., Fig. 6), it makes the implicit assumption that the orthogonal stimuli activate only complementary parts of the cortex. This should not be taken for granted. In fact, such an image enhancement approach can actually be justified only a posteriori, after single-condition maps have been thoroughly evaluated and minimal overlap of responses to the different stimuli has been demonstrated.

Single-condition maps were first obtained by Ts'o et al. (1990) and led to the optical imaging of the thin stripes in V2 of the macaque monkey. However, in previous studies of the organization of orientation preference single-condition maps were not presented (Blasdel and Salama, 1986; Ts'o et al., 1990), probably due to the limited signal-to-noise ratio of the orientation preference maps in the monkey. In the meantime, however, there have been technical improvements (Ratzlaff and Grinvald, 1991) that have considerably improved the quality of the optical data. 

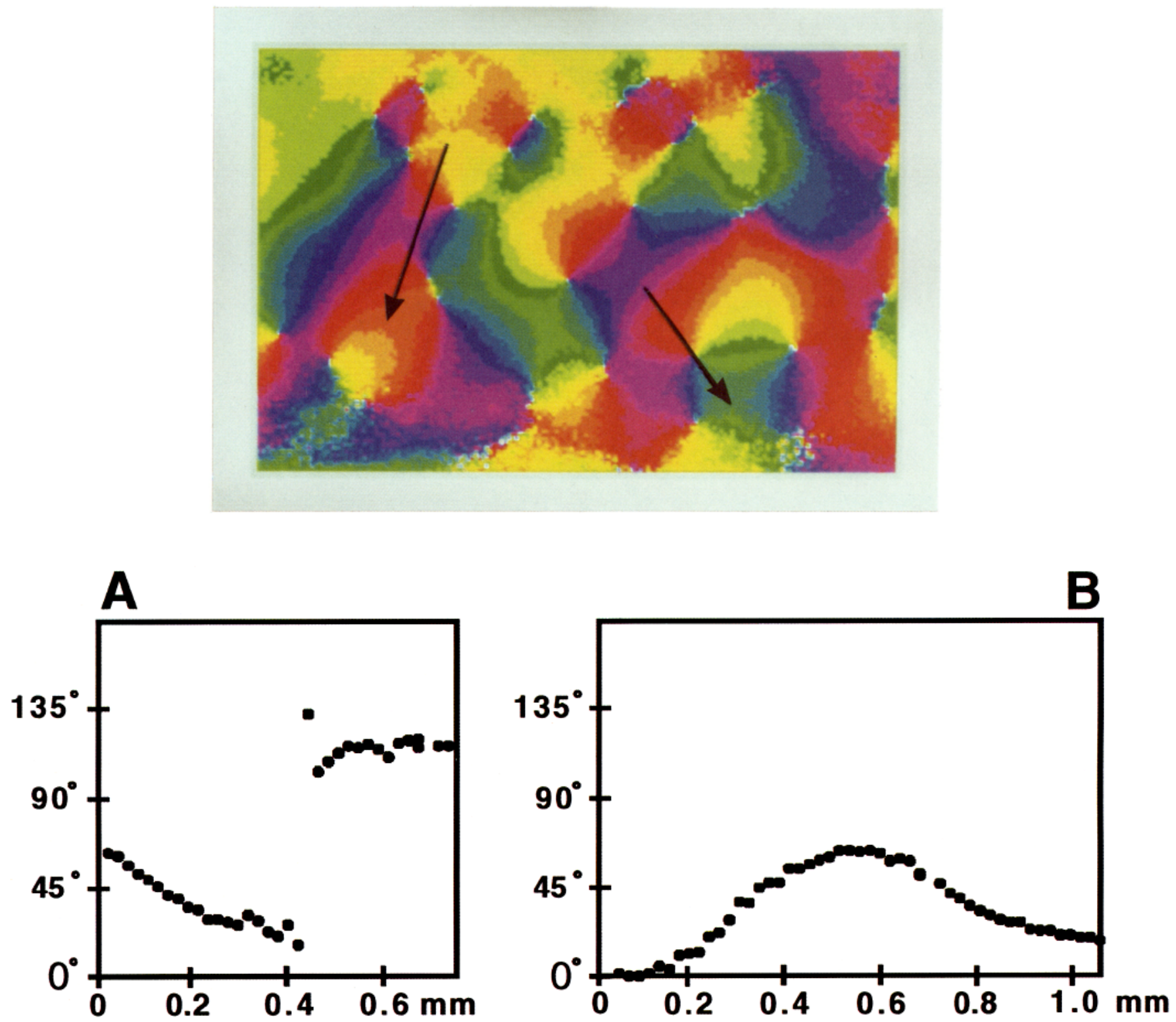

Figure 15. Computed "tangential penetrations" through an orientation preference map. The arrows on the upper image show the selected tracks used. The plots in the bottom part are plots of orientation (ordinate, in degrees) versus track distance (abscissa, in millimeters). $A$ shows the preferred orientations recorded along path $\mathrm{A}$, which traverses a singularity. The plot clearly shows the $90^{\circ}$ jump in orientation preference. Track $B$ is taken from a path lying next to a clockwise and a counterclockwise pinwheel. The resulting plot shows a curve composed of two components, first the ascending (passing the clockwise pinwheel) and then the descending one (passing the counterclockwise pinwheel).

\section{Directionality columns}

The experiments presented in this report show that the maps obtained with a grating moving in one direction are similar to those obtained with gratings of identical orientations but moving in the opposite direction (Fig. $7 C$ ). This suggests that cells in cat area 18 are not well segregated into directionality columns. Our result is at variance with a report of Swindale et al. (1987), who reported existence of clusters of cells with similar direction preferences for cat area 18 . The clusters described had a size of the order of $500 \mu \mathrm{m}$ and should thus be easily resolvable with intrinsic optical imaging. In none of our experiments were we able to find such direction-selective regions in area 18. One remaining possibility is that clusters of cells with opposite direction preference lie exactly above each other. In such a case, optical imaging may not be in a position to resolve direction- selective clusters because of its limited depth resolution. This explanation is, however, unlikely. An article by Tolhurst et al. (1981) reports-albeit in area 17-that direction preferences are similar for cells across one whole cortical column reaching from the pia to the white matter. Furthermore, 2-DG studies show that patterns obtained with a stimulus moving in only one direction span all cortical layers (S. Löwel, personal communication). We therefore propose that cells in area 18 are at least not strongly clustered with regard to direction selectivity.

\section{Bands or patches?}

2-DG pictures published in the literature seem to suggest that regions preferring the same orientation are elongated bands or slabs rather than patches (see, e.g., Hubel et al., 1977, 1978; Singer, 1981, Albus and Sieber, 1984; Löwel et al., 1987). Indeed, Figures $2-4$ and $6-8$ of our data, obtained by optical 


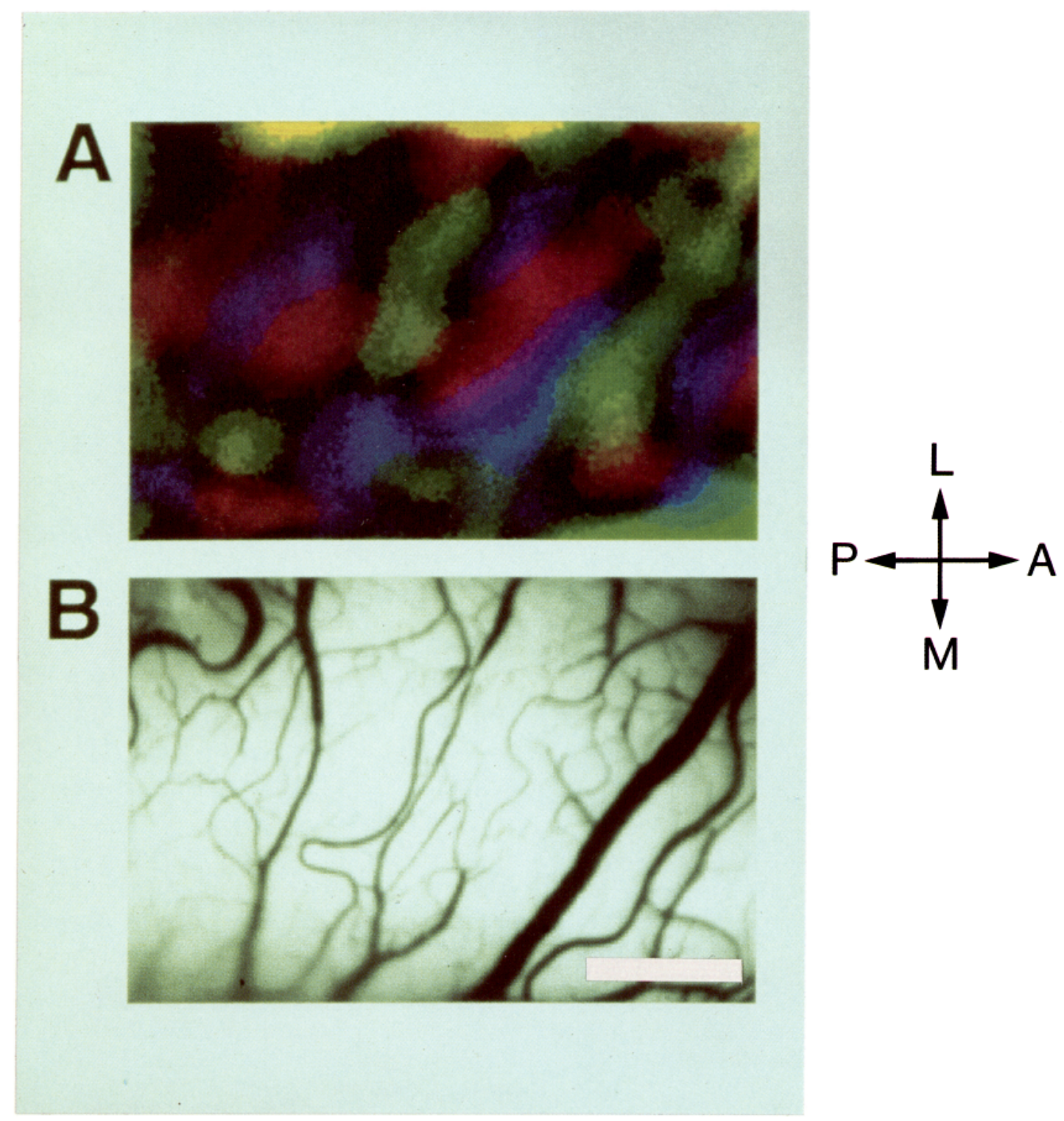

Figure 16. Polar maps. A, In this map two different types of information are combined: colors code for the preferred orientations, and the brightness stands for the sharpness of orientation tuning (with the limitations explained in the text). Areas with very narrow tuning curves show light colors, whereas areas without strong orientation preferences or with weak responses appear dark (e.g., orientation centers). $B$ shows the vascular pattern of the cortical area mapped in $A$. Scale bar, $1 \mathrm{~mm}$.

imaging, also seem to forward such an interpretation. However, if one surveys at the organization of orientation preferences for all the orientations (which is not possible with the 2-DG technique) the picture is different. This can be seen in Figure 8, which shows four single-condition maps having a beaded appearance similar to that previously described in 2-DG experiments (Löwel et al., 1987). Figure $8 C$, for example, shows a banded structure with three "beads." However, close inspection of the data shows that the gray areas between the bands are often best activated by $22.5^{\circ}$ or $45^{\circ}$ different orientations. Some of the dark patches in Figure $8 B$, for example, are found in locations that are gray in Figure $8 C$.

In tree shrews microelectrode recordings (e.g., Humphrey and Norton, 1980) and 2-DG experiments (Humphrey et al., 1980) have been used to determine the pattern of iso-orientation domains. It has been proposed that iso-orientation domains in this species constitute bands, and in fact the 2-DG maps in Hum- phrey et al. (1980) look very banded, certainly more banded than the published pictures in cat area 17 or area 18 . One may wonder whether in these animals the organization of orientation preference could be different from the cat. Two facts suggest that this might indeed be the case. In carefully looking at the microelectrode recordings from tree shrew striate cortex (e.g., Humphrey and Norton, 1980), one observes that the discontinuities $\left(90^{\circ}\right.$ jumps) in orientation preference that have been reported in the cat are missing in this animal. We have shown above that these discontinuities in cat are likely to be the manifestations of orientation centers in microelectrode recordings. Therefore, if these sudden breaks are indeed not present in the tree shrew, it suggests that the organization of iso-orientation domains in tree shrews is markedly different from the cat. Second, it has been shown that tree shrews do not posses any ocular dominance columns; in these animals ocular dominance is organized in layers (Muly and Fitzpatrick, 1992). Therefore, since 
Figure 17. Orientation gradient maps. $A$, Using a two-dimensional gradient operator, the rate of orientation change for every point in the picture was determined. The resulting values are displayed on the map using a gray scale. White stands for a strong orientation change. Note that the orientation centers exhibit the strongest gradients and that there are no lines of rapid orientation change ("fractures") elsewhere. $B$ shows the vascular pattern corresponding to the map in $A$. Scale bar, $1 \mathrm{~mm}$.
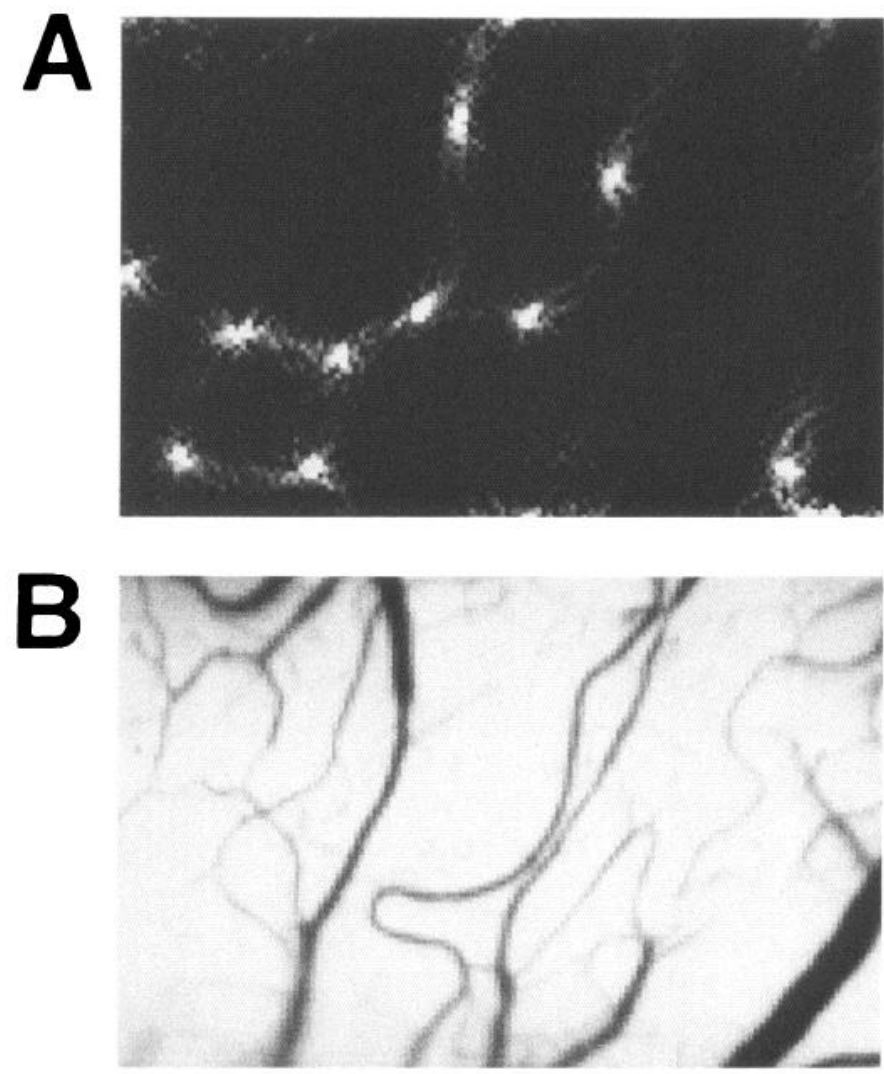

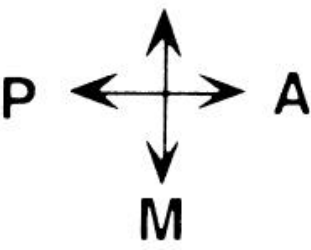

\section{Continuity of pinwheels around orientation centers}

We observed that the patches of iso-orientation domains group around "orientation centers." In these small regions all orientation preferences border onto one another. This is illustrated in Figure 20, which shows a single-condition map obtained with one particular grating. On this map we overlaid the outline of the most strongly activated patches with blue. In addition, we superimposed the outlines obtained from three other singlecondition maps obtained with the remaining three stimulus orientations. A star is placed in locations where we detected a pinwheel center in the angle map. This picture then illustrates how combining more maps can lead to pinwheels and orientation centers, even though a single picture still has the banded appearance: some regions (grayish areas) that show an intermediate response in the black-and-white activity map-and might therefore be considered belonging to a band connecting two dark beads - turn out to be very strongly activated by another orientation. One can observe that outlines with different colors lie over these grayish areas. These outlines group around the center marked with the star, thereby constituting the surrounding pinwheel.

Figure 20 shows how the data that led us to conclude that iso-orientation domains are radially organized in a pinwheellike fashion rather than in bands are not at variance with data from 2-DG studies. It is rather that richer data enabled us to gain a more complete picture of the overall organization. Furthermore, the continuous representation of orientation preference in pinwheels (Fig. 20 C) is fully consistent with the smooth changes of preferred orientation observed in long tangential penetrations.

The regions exhibiting different orientation preferences are
Figure 18. Distributions of iso-orientation domains in area 18. Each bar shows the amount of cortical area devoted to the processing of a given orientation (with a resolution of $22.5^{\circ}$ ). The distribution is nearly flat, indicating that all orientations are equally represented in areas 18 . 

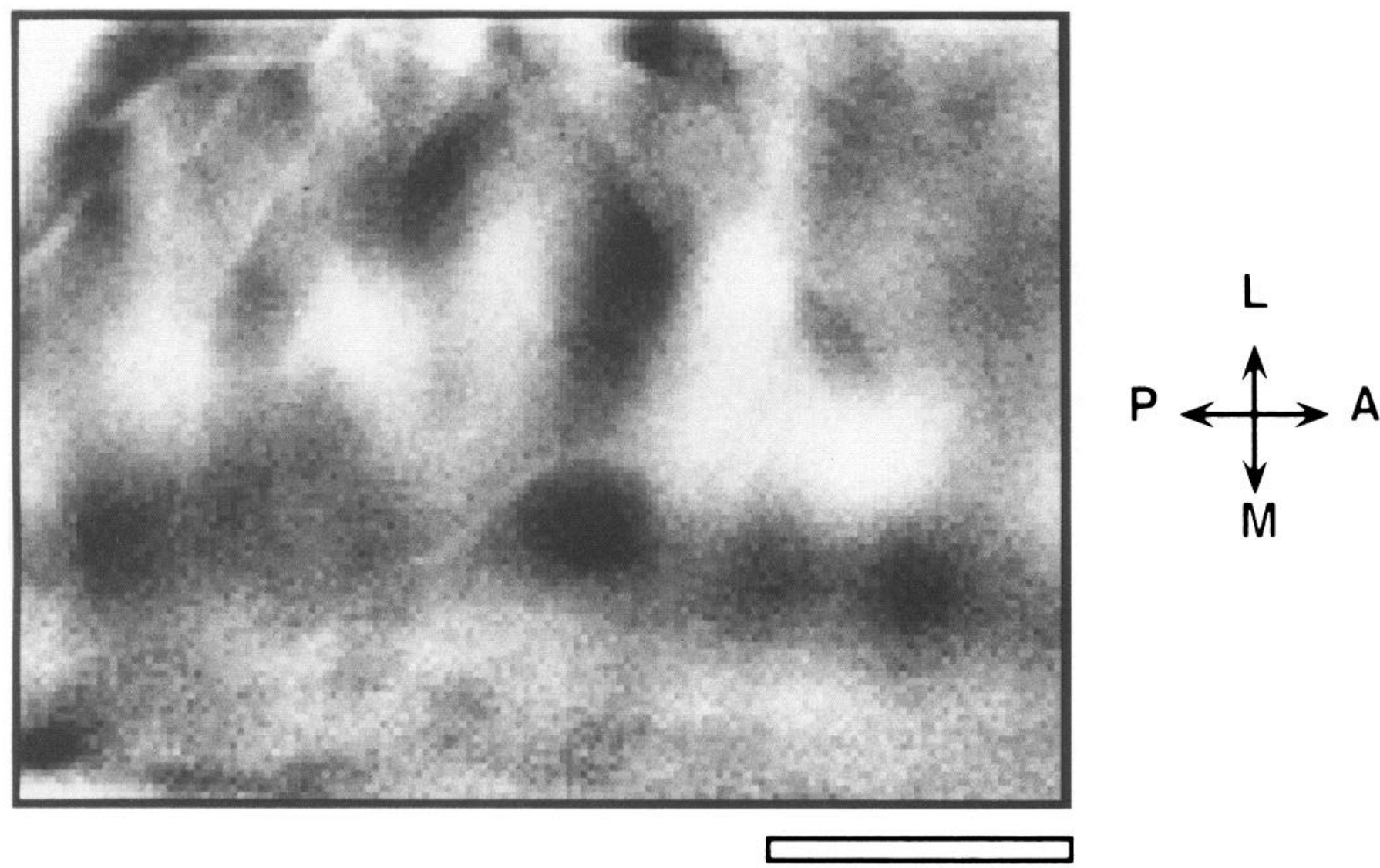

Figure 19. Clustering of cells with selective response properties other than orientation in area 18. The map shows the activation of cortex by the complete stimulus set used here. First, all eight orientations were added and then the sum was divided by the genuine blank. This map shows dark activation area of a patchy nature, rather than a uniform activation of the entire cortical region. The amplitude of this map is approximately $30 \%$ of the amplitude of the different single-condition maps. This result suggests that there are receptive-field properties other than orientation that may selectively activate the lighter regions in this map. Each stimulus was averaged 96 times. Scale bar, $1 \mathrm{~mm}$.

distributed around the centers either in a clockwise or a counterclockwise fashion. Centers of this sort have also been observed by Swindale et al. (1987) using maps obtained from interpolation between multiple electrode recordings. Our findings corroborate and extend this study, since we are able to obtain continuous data from every site in the imaged cortical area with our method. This is desirable for a precise mapping of the orientation centers - a difficult task with multiple singleunit recordings.

In the monkey cortex it seems natural to assume (Götz, 1987) that the orientation centers would come to lie in the cytochrome oxidase-rich "blobs" (Horton and Hubel, 1981), which are known to contain many cells exhibiting a poor orientation tuning (Livingstone and Hubel, 1984). Recently cytochrome oxidase patches were also reported in cat area 17 (Murphy et al., 1990). These patches are, however, very weak or absent in area 18 (K. Murphy, personal communication), so it seems unlikely that cytochrome oxidase patches in the cat could correspond to pinwheel centers.

\section{One or two orientation cycles?}

It has been suggested (e.g., von Seelen, 1970; Braitenberg and Braitenberg, 1979) that the visual cortex could be organized around orientation centers instead of being a banded structure as proposed by Hubel and Wiesel in their "ice-cube" model (Hubel and Wiesel, 1977). Braitenberg and Braitenberg (1979) showed that much of the data obtained by Hubel and Wiesel could very well fit into a scheme of circular organization. Braitenberg and Braitenberg proposed furthermore that these centers should be spaced at a distance of approximately $0.5 \mathrm{~mm}$ and that every orientation should appear twice around an orientation center. More recently, different groups have proposed, mostly on theoretical grounds, an organization with orientation centers around which every orientation appears once (Swindale, 1982; Linsker, 1986; Baxter and Dow, 1989; Obermayer et al., 1990; Miller, 1992). Götz (1987) was the first to examine closely the experimental data of Blasdel and Salama (1986) for orientational singularities and found that in monkey V1 there are indeed orientation centers around which every orientation appears once. Some of these authors (Swindale, 1982; Götz, 1987) used the index of the rotational singularities to describe the different models. Singularities around which every orientation between $0^{\circ}$ and $180^{\circ}$ appears once have an index of \pm 0.5 ; orientation centers around which every orientation appears twice (i.e., $360^{\circ}$ orientation change appears once in the $360^{\circ}$ around the singularity) consequently have a index of \pm 1 (Braitenberg and Braitenberg model).

The index of the pinwheels is the crucial distinction between the models. We therefore inspected our data very closely with regard to the question whether the majority of our pinwheel centers have an index of \pm 0.5 or of \pm 1 . We found that all of the unequivocally identified pinwheel centers were of the type 

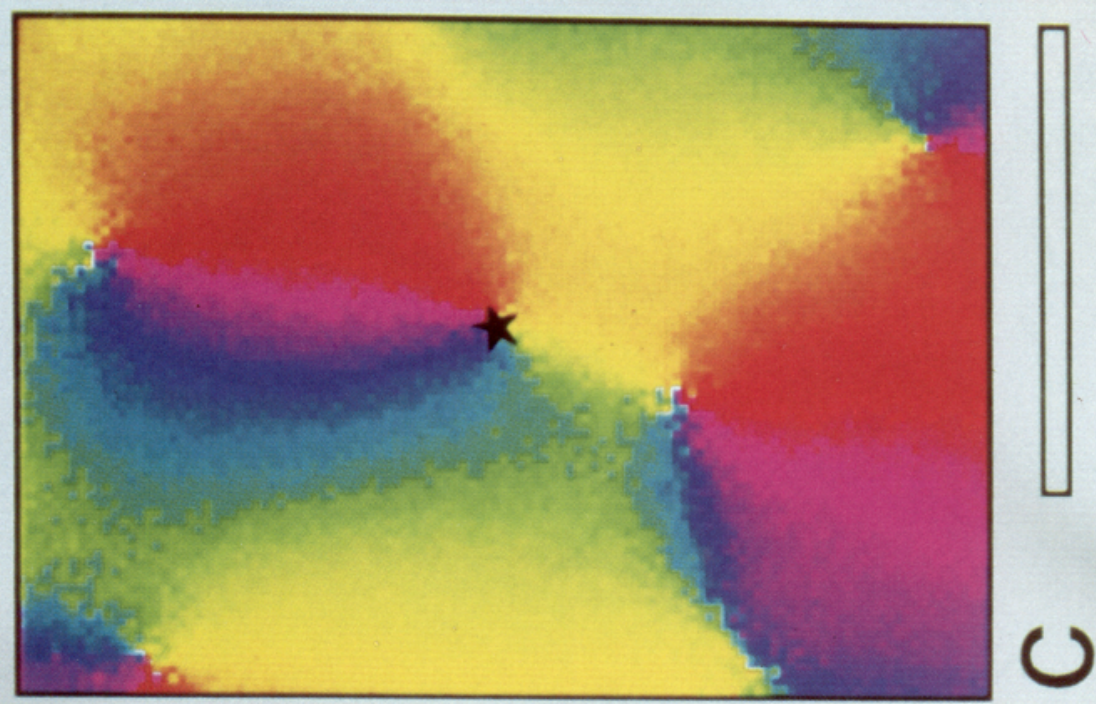

융.

9 \%

50.

8.

管

뿡은

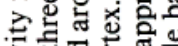

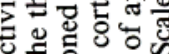

政

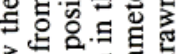

3.

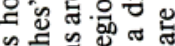

政.

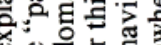

원형

눈.

啊

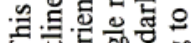

者

至

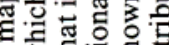

3

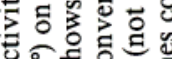

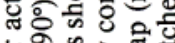

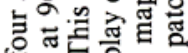

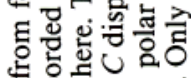

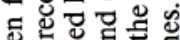

政

\%

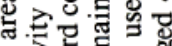

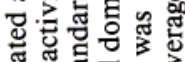

政

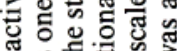

o 0

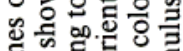

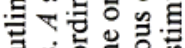

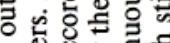

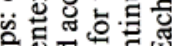

呵证

ㄷㅎㅇ응

달

7 .

당 뭉

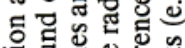

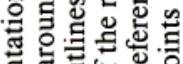

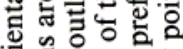

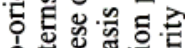

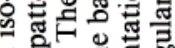
5 है o 일

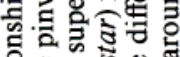
웡

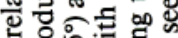

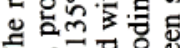
$\checkmark$ g

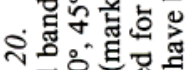
旸

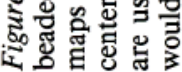


+0.5 . From this it is clear that theories proposing orientation centers with only one representation of all orientations around the center singularity are those that are in best accordance with the experimental observations.

\section{Polar maps and the spatial resolution of optical imaging}

Polar maps enable us to take a closer look at the organization of orientation in the investigated areas since they combine information about two parameters in one map. While angle maps only provide the angle value of the preferred orientation for each cortical location, polar maps additionally show how well oriented these regions are. Other types of maps such as maps of orientation tuning strength, or "HLS maps," can be derived from the optical data presented here. Such maps that can yield additional information are provided and discussed in a forthcoming article (T. Bonhoeffer, D.-S. Kim, D. Malonek, D. Shoham, and A. Grinvald, unpublished observations).

Although the interpretation of polar maps is straightforward in most cortical locations, in some areas, especially in regions of rapid orientation changes (e.g., in orientation centers), these maps have to be interpreted with caution. In order to clarify this issue, let us consider some methodological aspects of optical imaging based on intrinsic signals. The spatial resolution of optical imaging using intrinsic signals is determined by two different factors, the "biological" and the "optical" resolution. First, we determined whether the optical resolution of our apparatus was the limiting factor responsible for the observation of apparently poor orientation tuning at the pinwheel centers. We compared the polar maps obtained using lenses with different apertures and found that the sizc of the dark rcgion observed at the orientation centers remained constant, regardless which numerical aperture was used. This observation can serve as an indication that the dark spots at the orientation centers are not an artifact of the resolution limit of the optics.

Apart from the optical resolution, there is a biological resolution limit, namely, the best resolution that can be achieved using a signal originating from the microcirculation (Grinvald et al., 1986; Frostig et al., 1990). It is known that neurons in the mammalian cortex are never more than $50 \mu \mathrm{m}$ away from a microcapillary (Scharrer, 1944; Kuffler et al., 1984). Moreover, since we are integrating signals in the vertical direction over at least $500 \mu \mathrm{m}$, the signals from a "minicolumn" of $50 \times 50 \times$ $500 \mu \mathrm{m}^{3}$ originating from approximately 10 capillaries. Therefore, the capillary density is probably not a limiting factor for resolution of the technique. However, light-scattering properties of the tissue must also be considered. Orbach and Cohen (1983) showed that, in the salamander olfactory bulb, image blur due to light scattering and out-of-focus contributions was less than $200 \mu \mathrm{m}$ from a depth of $1 \mathrm{~mm}$. Preliminary measurements (D. Malonek and A. Grinvald, unpublished observations) suggest a similar value for the mammalian cortex.

Furthermore, it should be emphasized that whenever many maps are analytically compared, an improved resolution is achieved. It has been shown by simple simulations that, if the light scattering of the tissue is approximated by a Gaussian curve, two points cannot be resolved if they are closer than the half-width of the light-scattering curve. However, if a differential map is calculated, the spatial resolution increases and is mostly limited by the signal-to-noise ratio of the mapping signal (D. Shoham and A. Grinvald, personal communication). In conclusion, our current estimate is that in the configuration used here the technique has a (lateral) spatial resolution of 50-100 $\mu \mathrm{m}$.

This value then influences the interpretation of polar maps. In a location where different orientation preferences border onto each other, one is bound to find a broad orientation tuning, since locations from $\sim 50 \mu \mathrm{m}$ away (which, close to orientation centers, will have a very different preferred orientation) influence the signal at the recording site. Therefore, the fact that polar maps show dark spots in the orientation centers can not be taken as a direct evidence that orientation tuning is broad in these locations.

\section{"Tangential penetrations" and fractures in orientation maps}

One way of comparing our data with conventional electrophysiological data is to compute "tangential penetrations" in the optically recorded orientation preference maps. Many of the data obtained from long tangential penetrations are plotted as the preferred orientation of cells against the distance in the tangential penetration (Hubel and Wiesel, 1974; Albus, 1975, 1985). Many of these penetrations show the preferred orientation either changing smoothly with distance, in a clockwise or a counterclockwise fashion, or changing from counterclockwise to clockwise and vice versa. In a few cases, however, jumps in orientation preference occur, usually of $90^{\circ}$. Figure 15 shows how very similar "tracks" were obtained from the optical data. We inspected gradient maps in which the rate of orientation change was calculated for additional locations where jumps could occur. These maps show that in cat area 18 there are no additional lines or fractures where orientation changes rapidly (Fig. 17). From our data we therefore conclude that the $90^{\circ}$ jumps in orientation preference that are observed in tangential penetrations are due to the electrode passing through an orientation center.

These data are at variance with the reports of Blasdel and coworkers (Blasdel and Salama, 1986; Blasdel, 1992a,b), who have reported that there are numerous lines of rapid orientation changes or fractures in monkey V1. The most obvious possible differences between our and their data are the different species and the different visual areas examined. Their experiments were carried out in monkey $\mathrm{Vl}$, ours in cat area 18 . A further possibility, however, is that the different interpretation is only a matter of scale. Also in our data one can recognize a very slightly enhanced rate of orientation change along some lines that manifest themselves as slightly grayish lines (see Fig. 17). If these are the structures that Blasdel and Salama report as having a high rate of orientation change, then we too see fractures. However, the rate of orientation change at orientation centers is $\sim 3600^{\circ} / \mathrm{mm}$ whereas in the faint gray lines it was only $\sim 300$ $400 \% \mathrm{~mm}$, and in the rest of the cortex $\sim 70-180 \% \mathrm{~mm}$. These numbers indicate that the values for the orientation centers are radically different, whereas the data on the grayish lines are only slightly different from the rest of the cortex.

It is interesting to compare our results with those of Blasdel (Blasdel and Salama, 1986; Blasdel, 1989) with respect to the organization of iso-orientation domains, that is, the pinwheel organization. Blasdel (1992a) remarked recently that the data of Blasdel and Salama (1986) clearly contained pinwheel-like structures. These were, however, pointed out only later by Götz (1987). Furthermore, Blasdel's model for the organization of iso-orientation domains that emerged from these pioneering experiments shows parallel bands over short distances as the key feature organizing the visual cortex. This model (Blasdel, 
1989) is inconsistent with the pinwheel structure shown in the present report. Another difference is that they propose that fractures parcelate the visual cortex to delineate modules that could then be the subunits of visual cortical processing. The fact that these fractures do not exist in cat visual cortex (while pinwheels do) obviously does not disprove this idea. On the other hand, the lack of fractures in a different species makes it less likely that fractures are an important feature underlying the organization of the visual cortex. In fact, more recent studies of monkey striate cortex by Bartfeld and Grinvald (1992) suggest that the architecture of the orientation domain is nearly identical in the cat and the monkey.

\section{Random organization of iso-orientation domains versus pinwheel organization}

Schwartz and Rojer (1992) have shown that smoothing of a random noise pattern can cause the appearance of orientation patches. We have obtained similar results in our own simulations. There is, however, one significant difference between the experiments and the simulations: the random noise patterns are not reproducible from one simulation to the next whereas the experimental results are highly reproduciblc (c.g., Figs. 4, 5). Furthermore, as already mentioned, in other studies orientation patches detected optically were confirmed by electrical recordings or by $2-\mathrm{DG}$ imaging, both techniques that have excellent spatial resolution.

It has also been pointed out that the presence of pinwheels is a consequence of a basic topological theorem; mapping of a circular function like orientation onto two dimensions automatically creates a map with pinwheels (Schwartz and Rojer, 1992). Thus, a critical question is whether the pinwheel patterns are a "topological artifact" produced by noisy data and smoothing, or whether they indeed reflect the radial organization of iso-orientation domains claimed here. Our own simulations using random sets of "orientation patches" yielded "angle maps" that were, of course, consistent with the theorem and similar to the artificial pinwheel images shown by Schwartz. However, again wc notcd a significant difference between the experimental data and the simulations. The polar maps resulting from the simulation of random orientation patterns exhibited long lines and many loops of low magnitude (D. Malonek, D. Shoham, and A. Grinvald, personal communication). This indicates that in these simulations iso-orientation patches are not organized in a truly radial fashion around points of singularity. It is therefore in sharp contrast to the experimental polar maps, which only showed spotlike regions of low magnitude (e.g., Fig. 17). We cannot completely rule out the possibility that further simulations using different parameters or other assumptions would yield polar maps that are more similar to the experimental results. Nevertheless, we believe that the radial arrangement is real: the example in Figure 20 clearly indicates that iso-orientation domains are arranged in a radial fashion around orientation centers. This particular arrangement of iso-orientation domains was dominant in our data; only a small fraction of the iso-orientation domains (an example can be seen in the left part of the map in Fig. 15) showed a linear arrangement of rather short (1-2 $\mathrm{mm})$, parallel bands.

\section{The distribution of orientation processing in the cortex}

In their original work in the cat Hubel and Wiesel (1962) found a uniform distribution of preferred orientations. Later some authors proposed, at least for cat area 17, that the distribution of orientation-selective cells is nonuniform (Payne and Berman, 1983; Schall et al., 1986; Berman et al., 1987). The data presented in this study (Fig. 18) have the advantage that the sampling bias - a concern in all previous studies - is less of a problem here since the whole cortical surface within a given region is sampled. Our data suggest that the distribution of orientation preferences is rather flat in area $18\left(\chi^{2}=0.98,7 \mathrm{df}, p<0.01\right)$. This statement can, however, only be made for cortical regions that are accessible to optical method, that is, the part of area 18 that is on the lateral gyrus, corresponding to eccentricities from $0^{\circ}$ to $40^{\circ}$ from the vertical meridian and elevation of $-5^{\circ}$ to $-30^{\circ}$.

\section{Concluding remarks}

The pattern that emerges for the functional architecture in cat area 18 is the following. Direction selectivity is not an important parameter according to which neurons in area 18 are grouped together. Stimulating a cortex with gratings of different orientations and directions shows that opposite directions of movement (with the same orientation) activate the same regions in the cortex to a nearly equal extent whereas orthogonal orientations result in almost complementary iso-oricntation maps. These maps, when combined, show that iso-orientation domains are not elongated bands. Domains for all the orientations are grouped together around orientation centers in a pinwheellike fashion. The preferred orientations change smoothly across the cortical surface, the only exception being the centers of the pinwheels. In general, the structure of these maps-similar to what has been described for monkey V1 (Bartfeld and Grinvald, 1992) - is best characterized by the notion of "little long-range but great short-range order" as it was put by one of the manuscript referees.

It remains to be seen whether this organization serves an important function. One possibility is that the pinwheels and orientation centers we observe are merely produced because of the need to map a multidimensional space, consisting of retinotopic position, ocularity, and orientation preference onto the two-dimensional cortical surface (Durbin and Mitchison, 1990). It is, however, also possible that the nervous system makes functional use of this unique arrangement. In order to investigate this, it is important to know whether neurons situated at the orientation centers have particular functional properties, or a particular afferent or efferent connectivity. Furthermore, it would be very interesting to observe the development of this peculiar map during the course of the early life of a young kitten. Answers to these questions will hopefully contribute toward a further understanding of the importance of this meticulous organization for cortical development and function.

\section{Note added in proof:}

Functional organization for direction selectivity has recently been demonstrated in cat area 18, using optical imaging. Consistent with this study, the amplitude of the direction selectivity maps was two to three times weaker than that observed for orientation maps in the same cortical region (A. Shmuel, A. Arieli, D. Malonek, and A. Grinvald, personal communication).

\section{References}

Albus K (1975) A quantitative study of the projection area of the central and paracentral visual field in area 17 of the cat. II. The spatial organization of the orientation domain. Fxp Brain Res 24:181-202.

Albus K (1985) A microelectrode study of the spatial arrangement of iso-orientation bands in the cat's striate cortex. In: Models of the 
visual cortex (Rose D, Dobson VG, eds), pp 485-491. Chichester: Wiley.

Albus K, Sieber B (1984) On the spatial arrangement of iso-orientation bands in the cat's visual cortical areas 17 and 18: a ${ }^{14} \mathrm{C}$-deoxyglucose study. Exp Brain Res 56:384-388.

Bartfeld E, Grinvald A (1992) Relationships between orientation preference pinwheels, cytochrome oxidase blobs and ocular dominance columns in primate striate cortex. Proc Natl Acad Sci USA 89:1 190511909.

Baxter WT, Dow BM (1989) Horizontal organization of orientationsensitive cells in primate visual cortex. Biol Cybern 61:171-182.

Berman NEJ, Wilkes ME, Payne BR (1987) Organization of orientation and direction selectivity in areas 17 and 18 of cat cerebral cortex. J Neurophysiol 58:676-699.

Blasdel GG (1989) Topography of visual function as shown with voltage sensitive dyes. In: Sensory systems in the mammalian brain (Lund JS, ed), pp 242-268. New York: Oxford UP.

Blasdel GG (1992a) Differential imaging of ocular dominance and orientation selectivity in monkey striate cortex. J Neurosci 12:31153138.

Blasdel GG (1992b) Orientation selectivity, preference, and continuity in monkey striate cortex. J Neurosci 12:3139-3161.

Blasdel GG, Salama G (1986) Voltage-sensitive dyes reveal a modular organization in monkey striate cortex. Nature 321:579-585.

Bonhoeffer T, Grinvald A (1991) Iso-orientation domains in cat visual cortex are arranged in pinwheel-like patterns. Nature 353:429-431.

Braitenberg V, Braitenberg C (1979) Geometry of orientation columns in the visual cortex. Biol Cybern 33:179-186.

Durbin R, Mitchison G (1990) A dimension reduction framework for understanding cortical maps. Nature 343:644-647.

Friedman HR, Bruce CJ, Goldman-Rakic PS (1987) A sequential double-label ${ }^{14} \mathrm{C}$ - and ${ }^{3} \mathrm{H}-2-\mathrm{DG}$ technique. Validation by double-dissociation of functional states. Exp Brain Res 66:543-554.

Frostig RD, Lieke EE, Ts'o DY, Grinvald A (1990) Cortical functional architecture and local coupling between neuronal activity and the microcirculation revealed by in vivo high-resolution optical imaging of intrinsic signals. Proc Natl Acad Sci USA 87:6082-6086.

Gilbert CD, Wiesel TN (1990) The influence of contextual stimuli on the orientation selectivity of cells in primary visual cortex of the cat. Vision Res 30:1689-1701.

Götz KG (1987) Do "d-blob" and "l-blob" hypercolumns tessellate the monkey visual cortex. Biol Cybern 56:107-109.

Grinvald A, Anglister L, Freeman JA, Hildesheim R, Manker A (1984) Real-time optical imaging of naturally evoked electrical activity in intact frog brain. Nature 308:848-850.

Grinvald A, Lieke E, Frostig RD, Gilbert CD, Wiesel TN (1986) Functional architecture of cortex revealed by optical imaging of intrinsic signals. Nature 324:361-364.

Grinvald A, Frostig RD, Siegel RM, Bartfeld E (1991a) High-resolution optical imaging of functional brain architecture in the awake monkey. Proc Natl Acad Sci USA 88:11559-11563.

Grinvald A, Bonhoeffer T, Malonck D, Shoham D, Bartfeld E, Aricli A, Hildesheim R, RatzlaffE (1991b) Optical imaging of architecture and function in the living brain. In: Memory: organization and locus of change (Squire LR, Weinberger NM, Lynch G, McGaugh JL, eds), pp 49-88. New York: Oxford UP.

Horton JC, Hubel DH (i981) Regular patchy distribution of cytochrome oxidase staining in primary visual cortex of macaque monkey. Nature 292:762-764.

Hubel DH, Wiesel TN (1962) Receptive fields, binocular interaction and functional architecture in the cat's visual cortex. J Physiol (Lond) 160:106-154

Hubel DH, Wiesel TN (1963) Shape and arrangement of columns in cat's striate cortex. J Physiol (Lond) 160:106-154.

Hubel DH, Wiesel TN (1965) Receptive fields and functional architecture in two non-striate visual areas (18 and 19) of the cat. J Neurophysiol 28:229-289.

Hubel DH, Wiesel TN (1974) Sequence regularity and geometry of orientation columns in the monkey striate cortex. J Comp Neurol 158:267-293.

Hubel DH, Wiesel TN (1977) Ferrier lecture. Functional architecture of macaque monkey visual cortex. Proc R Soc Lond [Biol] 198:1-59.

Hubel DH, Wiesel TN, Stryker MP (1977) Orientation columns in macaque monkey visual cortex demonstrated by the 2-deoxyglucose autoradiographic technique. Nature 269:328-330.

Hubel DH, Wiesel TN, Stryker MP (1978) Anatomical demonstration of orientation columns in macaque monkey. J Comp Neurol 177: 361-380.

Humphrey AL, Norton TT (1980) Topographic organization of the orientation column system in the striate cortex of the tree shrew (Tupaia glis). I. Microelectrode recording. J Comp Neurol 192:531547.

Humphrey AL, Skeen LC, Norton TT (1980) Topographic organization of the orientation column system in the striate cortex of tree shrew (Tupaia glis). II. Deoxyglucose mapping. J Comp Neurol 192: 549-566.

Kaas JH, Nelson RJ, Sur M, Lin C-S, Merzenich MM (1979) Multiple representation of the body within the primary somatosensory cortex of primates. Science 204:521-523.

Kauer JS (1988) Real-time imaging of evoked activity in local circuits of the salamander olfactory bulb. Nature 331:166-168.

Kim JH, Dunn MB, Hua Y, Rydberg J, Yae H, Elias SA, Ebner TJ (1989) Imaging of cerebellar surface activation in vivo using voltage sensitive dyes. Neuroscience 31:613-623.

Kuffer SW, Nicholls JG, Martin AR (1984) From neuron to brain. Sunderland, MA: Sinauer.

Linsker R (1986) From basic network principles to neural architecture: emergence of orientation columns. Proc Natl Acad Sci USA 83:87798783.

Livingstone MS, Hubel DH (1981) Effects of sleep and arousal on the processing of visual information in the cat. Nature 291:554-561.

Livingstone MS, Hubel DH (1984) Anatomy and physiology of a color system in the primate visual cortex. J Neurosci 4:309-356.

London JA, Cohen LB, Wu J-Y (1989) Optical recordings of the cortical response to whisker stimulation before and after the addition of an epileptogenic agent. J Neurosci 9:2182-2190.

Löwel S, Freeman B, Singer W (1987) Topographic organization of the orientation column system in large flat-mounts of the cat visual cortex: a 2-deoxyglucose study. J Comp Neurol 255:401-415.

Malonek D, Shoham D, Ratzlaff E, Grinvald A (1990) In vivo three dimensional optical imaging of functional architecture in primate visual cortex. Soc Neurosci Abstr 16:130.4.

Miller KD (1992) Development of orientation columns via competition between on- and off-center inputs. Neuroreport 3:73-76.

Muly EC, Fitzpatrick D (1992) The morphological basis for binocular and $\mathrm{ON} / \mathrm{OFF}$ convergence in tree shrew striate cortex. J Neurosci 12: 1319-1334.

Murphy KM, Van Sluyters RC, Jones DG (1990) Cytochrome-oxidase activity in cat visual cortex: is it periodic? Soc Neurosci Abstr 16: 130.3 .

Obermayer K, Ritter H, Schulten K (1990) A principle for the formation of the spatial structure of cortical feature maps. Proc Natl Acad Sci USA 90:8245-8349.

Orbach HS, Cohen LB (1983) Optical monitoring of activity from many areas of the in vivo and in vitro salamander olfactory bulb: a new method for studying functional organization in the vertebrate central nervous system. J Neurosci 3:729-750.

Orbach HS, Cohen LB, Grinvald A (1985) Optical mapping of electrical activity in rat somatosensory and visual cortex. $\mathrm{J}$ Neurosci 5:1886-1895.

Payne BR, Berman N (1983) Functional organization of neurons in cat striate cortex: variations in preferred orientation and orientation selectivity with receptive-field type, ocular dominance, and location in visual field map. J Neurophysiol 49:1051-1073.

Payne BR, Berman N, Murphy EH (1980) Organization of direction preferences in cat visual cortex. Brain Res 211:445-450.

Ratzlaff EH, Grinvald A (1991) A tandem-lens epifluorescence macroscope: hundred-fold brightness advantage for wide-field imaging. J Neurosci Methods 36:127-137.

Redies C, Diksic M, Riml H (1990) Functional organization in the ferret visual cortex: a double-label 2-deoxyglucose study. J Neurosci 10:2791-2803.

Schall JD, Vitek DJ, Leventhal AG (1986) Retinal constraints on orientation specificity in cat visual cortex. J Neurosci $6: 823-836$

Scharrer E (1944) The blood vessels of the nervous tissue. Q Rev Biol 19:308-318.

Schwartz EL, Rojer A (1992) A computational study of cortical hypercolumns and the topology of random orientation maps. Soc Neurosci Abstr 18:313.11.

Singer W (1981) Topographic organization of orientation columns in the cat visual cortex. A deoxyglucose study. Exp Brain Res 44:431436. 
Sokoloff L (1977) Relation between physiological function and energy metabolism in the central nervous system. J Neurochem 19:13-26. Swindale NV (1982) A model for the formation of orientation columns. Proc R Soc Lond [Biol] 215:211-230.

Swindale NV, Matsubara JA, Cynader MS (1987) Surface organization of orientation and direction selectivity in cat area 18 . J Neurosci 7:1414-1427.

Tolhurst DJ, Dean AF, Thompson ID (1981) Preferred direction of movement as an element in the organization of cat visual cortex. Exp Brain Res 44:340-342.

Tootell RBH, Silverman MS, Hamilton SL, De Valois RL, Switkes E (1988) Functional anatomy of macaque striate cortex. III. Color. J Neurosci 8:1569-1593.
Ts'o DY, Frostig RD, Lieke EE, Grinvald A (1990) Functional organization of primate visual cortex revealed by high resolution optical imaging. Science 249:417-420.

Tusa RJ, Palmer LA, Rosenquist AC (1978) The retinotopic organization of cat area 17 (striate cortex) in the cat. J Comp Neurol 177 213-236.

Tusa RJ, Rosenquist AC, Palmer LA (1979) Retinotopic organization of areas 18 and 19 in the cat. J Comp Neurol 185:657-678.

von Seelen W (1970) Zur Informationsverarbeitung im visuellen System der Wirbeltiere. Kybernetik 7:89-106.

Wiesel TN, Hubel DH, Lam DM (1974) Autoradiographic demonstration of ocular-dominance columns in the monkey striate cortex by means of transneuronal transport. Brain Res 79:273-279. 